EPJ manuscript No.

(will be inserted by the editor)

\title{
Nonlinear mean field Fokker-Planck equations. Application to the chemotaxis of biological populations
}

\author{
P.H. Chavanis \\ ' Laboratoire de Physique Théorique, Université Paul Sabatier, 118 route de Narbonne 31062 Toulouse, France \\ e-mail: chavanis@irsamc.ups-tlse.fr \\ To be included later
}

\begin{abstract}
We study a general class of nonlinear mean field Fokker-Planck equations in relation with an effective generalized thermodynamical (E.G.T.) formalism. We show that these equations describe several physical systems such as: chemotaxis of bacterial populations, Bose-Einstein condensation in the canonical ensemble, porous media, generalized Cahn-Hilliard equations, Kuramoto model, BMF model, Burgers equation, Smoluchowski-Poisson system for self-gravitating Brownian particles, Debye-Hückel theory of electrolytes, two-dimensional turbulence... In particular, we show that nonlinear mean field Fokker-Planck equations can provide generalized Keller-Segel models for the chemotaxis of biological populations. As an example, we introduce a new model of chemotaxis incorporating both effects of anomalous diffusion and exclusion principle (volume filling). Therefore, the notion of generalized thermodynamics can have applications for concrete physical systems. We also consider nonlinear mean field Fokker-Planck equations in phase space and show the passage from the generalized Kramers equation to the generalized Smoluchowski equation in a strong friction limit. Our formalism is simple and illustrated by several explicit examples corresponding to Boltzmann, Tsallis, Fermi-Dirac and Bose-Einstein entropies among others.
\end{abstract}

PACS. 05.20.-y Classical statistical mechanics - 05.45.-a Nonlinear dynamics and nonlinear dynamical systems

\section{Introduction}

The study of Fokker-Planck equations (Fokker (1914) [1, Planck (1917) 2]) is of considerable interest in physics 'since the pioneering work of Einstein (1905) [3] on the Brownian motion. The simplest Fokker-Planck equation is the Smoluchowski (1915) 4 equation. This is a driftdiffusion equation describing the diffusion of particles in physical space in the presence of an external potential (e.g. the sedimentation of colloids in a gravitational field). A more general Fokker-Planck equation is the Kramers (1940) [5 equation, previously introduced by Klein (1921) [6], which takes into account inertial effects and describes the diffusion of particles in phase space when they experience a friction force. The Smoluchowski equation is recovered from the Kramers equation in a strong friction limit where inertial effects are negligible. In this sense, the Smoluchowski equation describes an overdamped evolution. These Fokker-Planck equations [7] are consistent with usual thermodynamics in the canonical ensemble. They monotonically decrease the Boltzmann free energy ( $H$-theorem) and relax towards the equilibrium Boltzmann distribution.

Recently, several researchers have tried to extend the usual concepts of thermodynamics and kinetic theory in order to describe complex systems that are characterized by non-Boltzmannian distributions. In that respect, some generalized forms of entropic functionals 1 have been introduced. One of the most popular "generalized entropy" is the Tsallis (1988) 9] entropy, but other entropies have been presented by Abe (1997) [10, Borges \& Roditi (1998) [11, Kaniadakis (2001) [12, Naudts (2004) [13, and Kaniadakis et al. (2005) [14. It was later realized that these entropic functionals are special cases of the one-parameter family of entropies introduced earlier by Harvda \& Charvat (1967) [15] or of the two-parameters family of entropies introduced by Mittal (1975) [16] and Sharma \& Taneja (1975) [17. Other famous forms of entropies have been presented by Reyni (1970) 18 and Sharma \& Mittal (1975) [19. We refer to Kaniadakis \& Lissia 20, for a very interesting discussion of these historical aspects, starting from the early works of Euler in 1779.

\footnotetext{
1 We recall that the Boltzmann entropy can be obtained from a combinatorial analysis assuming that all the accessible microstates are equiprobable. This is the basic postulate of statistical mechanics. Non-standard entropies can be relevant for complex systems where this postulate breaks down, i.e. when the accessible microstates are not equiprobable. This happens when the system prefers some regions of phase space better than others or when the particles are subjected to exclusion/inclusion principles or fine-grained constraints [8].
} 
P.H. Chavanis: Nonlinear mean field Fokker-Planck equations.

Following these developments, some researchers have tried to develop out-of-equilibrium theories associated to a generalized thermodynamical framework. In particular, it has been first shown by Plastino \& Plastino (1995) 21 that the Tsallis $q$-distributions are the steady states of a nonlinear Fokker-Planck equation taking into account anomalous diffusion. This type of equations had been previously considered by mathematicians to describe porous media 22. The seminal work of Plastino \& Plastino 21] has been further developed by Tsallis \& Bukman (1996) [23], Stariolo (1997) 24, Borland (1998) 25] and Nobre et al. (2004) [26] among others. On the other hand, Kaniadakis \& Quarati (1994) 27] have introduced nonlinear Fokker-Planck equations whose steady states are the Fermi-Dirac 2 and Bose-Einstein statistics. These kinetic equations take into account an exclusion (fermions) or inclusion (bosons) principle leading to quantum-like statistics at equilibrium. The case of intermediate statistics, interpolating between fermions and bosons, has also been considered in 27. Recently, the bosonic Kramers equation has been studied in 30] and was shown to reproduce the phenomenology of the Bose-Einstein condensation in the canonical ensemble.

The above-mentioned nonlinear Fokker-Planck (NFP) equations are associated with special forms of entropic functionals (Tsallis, Fermi-Dirac, Bose-Einstein). More recently, Martinez et al. (1998) 31, Kaniadakis (2001) 12, Frank (2002) 32 and Chavanis (2003) 33 have studied generalized forms of NFP equations associated with an almost arbitrary entropic functional. They can be viewed as generalized Kramers and Smoluchowski equations where the coefficients of diffusion, friction and drift explicitly depend on the local density of particles. Physically, this can take into account microscopic constraints (exclusion volume effects, steric hindrance, non-extensive effects...) that modify the dynamics of the particles at small scales and lead to non-standard equilibrium distributions 3 . Martinez et al. 31 determined the NFP equation in order to recover, as a steady state, the equilibrium state produced by minimizing a generalized form of free energy at fixed mass. Kaniadakis [12] obtained the NFP equation

\footnotetext{
2 A generalized Fokker-Planck equation leading to the FermiDirac statistics has also been introduced by Chavanis et al. (1996) 28 in the context of the violent relaxation of collisionless stellar systems described by the Vlasov equation. This is based on the Lynden-Bell's form of entropy (1968) 29] which becomes similar to the Fermi-Dirac entropy in the two-levels approximation of the theory.

3 Generalized Kramers and Smoluchowski equations describe dissipative systems where the temperature is fixed instead of the energy. They are therefore associated with the canonical ensemble. The appropriate thermodynamical potential is the free energy $F=E-T S$ which decreases monotonically with time at fixed mass. Generalized Boltzmann and Landau equations describing conservative systems where the energy is fixed have been introduced by Kaniadakis (2001) 12 and Chavanis (2004) 34. They are associated with the microcanonical ensemble. The proper thermodynamical potential is the entropy $S$ which increases monotonically with time at fixed mass and energy.
}

from the Master equation (see also Curado \& Nobre [35]) by allowing the transition probabilities to depend on the concentration of particles in the initial and arrival states. Frank 32 derived the NFP equation from a generalized free energy functional by using the linear thermodynamics of Onsager. Chavanis 33. obtained the NFP equation by using a form of Maximum Entropy Production Principle (MEPP). This corresponds to a variational version of the linear thermodynamics of Onsager. We refer to the book of Frank [36] for a first survey on nonlinear Fokker-Planck equations.

Another topic of active research in statistical physics concerns the dynamics and thermodynamics of systems with long-range interactions [37. Several Hamiltonian systems with long-range interactions have been studied in the microcanonical ensemble such as self-gravitating systems, two-dimensional vortices, the HMF model, the freeelectron laser etc... For such systems the mean field approximation provides a very good description of the system and becomes exact in a proper thermodynamic limit $N \rightarrow+\infty$. The microcanonical ensemble is the correct description of isolated systems evolving at fixed energy. On the other hand, some authors have introduced a canonical version of these models so as to treat systems that are dissipative. This leads to the notion of Brownian systems with long-range interactions. Their dynamics is described by mean field Fokker-Planck equations where the temperature is fixed (instead of the energy). These mean field Fokker-Planck equations were introduced early by Kuramoto (1984) 38 to describe the synchronization of globally coupled nonlinear oscillators and more recently by Marzel \& Aslangul (2001) 39, Chavanis (2006) 40 and Frank [36] in a more general context. Some specific studies have been made for self-gravitating Brownian particles 4142, and for the BMF model 43. which is the canonical version of the HMF model 44.

In view of the importance of these two topics: generalized thermodynamics and long-range interactions, we have introduced in [33] a class of nonlinear mean field FokkerPlanck equations (see Eq. (81) of [33]) that incorporate both a generalized free energy functional and a long-range potential of interaction. As an illustration, we studied with C. Sire a model of self-gravitating Langevin particles 45 (see also 46]) that combines self-gravity (long-range interactions) and anomalous diffusion (generalized thermodynamics) related to the Tsallis entropy and to the polytropic equation of state. In other words, this model couples the NFP equation introduced by Plastino \& Plastino 21 to the gravitational Poisson equation. Unfortunately, this model of self-gravitating Langevin particles has no clear application in astrophysics (because self-gravitating systems are generally not overdamped and not dissipative) and was introduced essentially as an interesting dynamical model with rich mathematical properties. However, it was noted in [45] that this model could have applications in unexpected area, like in the chemotaxis of bacterial populations...

The name chemotaxis refers to the motion of organisms induced by chemical signals [47. In some cases, the 
biological organisms (bacteria, amoebae, endothelial cells, ants...) secrete a substance (pheromone, smell, food, ...) that has an attractive effect on the organisms themselves. Therefore, in addition to their diffusive motion, they move preferentially along the gradient of concentration of the chemical they secrete (chemotactic flux). When attraction prevails over diffusion, the chemotaxis can trigger a self-accelerating process until a point at which aggregation takes place. This is the case for the slime mold Dictyostelium discoideum and for the bacteria Escherichia coli. This is referred to as chemotactic collapse. A model of slime mold aggregation has been introduced by Patlak (1953) 48] and Keller \& Segel (1971) 49] in the form of two coupled differential equations. The first equation is a drift-diffusion equation describing the evolution of the concentration of bacteria and the second equation is a diffusion equation with terms of source and degradation describing the evolution of the concentration of the chemical. In the simplest model, the diffusion coefficient $D$ and the mobility $\chi$ of the bacteria are constant. This forms the standard Keller-Segel model. However, the original Keller-Segel model allows these coefficients to depend on the concentration of the bacteria and of the chemical. The case where these coefficients depend on the concentration of the chemical $c(\mathbf{r}, t)$, but not on the concentration of bacteria $\rho(\mathbf{r}, t)$, has been considered by Othmer $\&$ Stevens 50. This leads to ordinary mean field FokkerPlanck equations (with respect to $\rho(\mathbf{r}, t)$ ) with space and time dependent coefficients. On the other hand, if we assume that the diffusion coefficient and the mobility of the bacteria depend on their concentration $\rho(\mathbf{r}, t)$, but not on the concentration $c(\mathbf{r}, t)$ of the secreted chemical, the original Keller-Segel model takes the form of a generalized mean field Fokker-Planck equation. Therefore, the KellerSegel model represents a fundamental example of nonlinear mean field Fokker-Planck equation with physical applications in biology, thereby justifying a notion of generalized thermodynamics. The analogy between the KellerSegel model and NFP equations was first pointed out in [33] and further developed in subsequent papers (see, e.g., [51]). This analogy makes possible to interprete results of chemotaxis in terms of nonlinear Fokker-Planck equations and generalized thermodynamics, which has not been considered so far by applied mathematicians working on this domain [52]. It thus allows to make a bridge between two different communities. In this analogy, the model of selfgravitating Langevin particles introduced by Chavanis \& Sire 45 also provides a generalized Keller-Segel model of chemotaxis taking into account anomalous diffusion (the application of this model to chemotaxis has been emphasized in 53]). More generally, we can use the numerous results accumulated in the context of generalized thermodynamics to propose new forms of generalized Keller-Segel models with potential applications in biology.

The aim of this paper is to develop a simple and rich formalism that allows to deal with nonlinear mean field Fokker-Planck equations. We shall illustrate this formalism on several examples and show the inter-connections between different topics. The paper is organized as follows.
In Sec. 2, we consider NFP equations in physical space. This corresponds to overdamped models where inertial effects are neglected. We review and complete the basic properties of these equations in relation with an effective generalized thermodynamical (E.G.T.) formalism. In Sec. 2.1. we show that they can be obtained from generalized Langevin equations. In Sec. 2.5. we show that they admit an $H$-theorem (in the canonical ensemble) for a generalized free energy. We stress that the Legendre structure of the free energy and the Einstein relation are preserved in this generalized thermodynamical framework. In Sec. 2.6. we determine the steady states of these nonlinear mean field Fokker-Planck equations and show that they are solutions of an integrodifferential equation. In Sec. 2.7 (and in Appendix B), we show that a steady state of a nonlinear mean field Fokker-Planck equation is linearly dynamically stable if and only if (iff) it is a (local) minimum of the free energy at fixed mass. In Sec. 2.9] we show that a NFP equation in physical space with a constant mobility and a density-dependent diffusion coefficient can be written in the form of a generalized Smoluchowski equation incorporating a barotropic equation of state. In Secs. 2.10 and 2.11] we show the correspondance between the phenomenological derivations of the NFP equations given by Frank 32 and Chavanis 33 and the kinetic derivation given by Kaniadakis [12]. In Sec. 3, we present several explicit examples of NFP equations and mention their potential applications to the problem of chemotaxis (see Sec. 2.4). In particular, we introduce a new model of chemotaxis that incorporates both effects of anomalous diffusion and exclusion principle (volume filling). The corresponding generalized entropy is expressed in the form of integrals that can be explicited in particular cases. In Sec. 4. we consider NFP equations in phase space taking into account inertial effects. In Secs. 4.1,4.7, we list their main properties. In Sec. 4.8 we consider the strong friction limit and derive the generalized Smoluchowski equation from the generalized Kramers equation. We use a method of moments that is simpler than the Chapman-Enskog expansion presented in [54. In Sec. 5] we consider explicit examples corresponding to the Boltzmann, Tsallis and Fermi-Dirac entropies. The Appendices contain important results that complete the basic properties of the NFP equations discussed in the text. In Appendix A, we show that a generalized isotropic BGK operator has properties similar to those possessed by a nonlinear Kramers operator. In Appendix B, we establish a simple relation showing the equivalence between linear dynamical stability (exponential damping of the perturbation) and generalized thermodynamical stability (minimum of free energy at fixed mass). In Appendix C, we study the stability of a spatially homogeneous solution of the nonlinear mean field Fokker-Planck equation and evidence a critical point. In Appendices $\mathrm{D}$ and $\mathrm{E}$ we show that a distribution function $f$ in phase space is a minimum of the free energy $F[f]$ at fixed mass iff the corresponding distribution $\rho$ in physical space is a minimum of the corresponding free energy $F[\rho]$ at fixed mass. This implies that a distribution function $f$ in phase space is a stable steady state of the generalized 
P.H. Chavanis: Nonlinear mean field Fokker-Planck equations.

Kramers equation iff the corresponding distribution $\rho$ in physical space is a stable steady state of the corresponding generalized Smoluchowski equation. In Appendix E we extend to $d$ dimensions the Eddington formula that allows to obtain the distribution function $f=f(\epsilon)$ in phase space from the knowledge of the barotropic equation of state $p=p(\rho)$ in physical space. In Appendix G we derive the $H$-theorems associated with the NFP equations and in Appendix $\mathrm{H}$ we derive the polytropic equation of state associated with the Tsallis statistics.

\section{Nonlinear mean field Fokker-Planck equations in physical space}

We first describe nonlinear mean field Fokker-Planck equations in physical space where the inertia of the particles is neglected. They can be viewed as models describing an overdamped dynamics.

\subsection{Generalized Langevin equations}

We consider a system of $N$ particles whose individual dynamics is described by the stochastic Ito-Langevin equations

$$
\frac{d \mathbf{r}_{i}}{d t}=-\chi\left(\rho_{i}\right) \nabla \Phi_{i}+\sqrt{2 D\left(\rho_{i}\right)} \mathbf{R}_{i}(t)
$$

where $\mathbf{R}_{i}(t)$ is a white noise satisfying $\left\langle\mathbf{R}_{i}(t)\right\rangle=\mathbf{0}$ and $\left\langle R_{i, \alpha}(t) R_{j, \beta}\left(t^{\prime}\right)\right\rangle=\delta_{i j} \delta_{\alpha, \beta} \delta\left(t-t^{\prime}\right)$ where $i=1, \ldots, N$ label the particles and $\alpha=1, \ldots, d$ label the coordinates of space. We have noted $\rho_{i}=\rho\left(\mathbf{r}_{i}(t), t\right)$ and $\Phi_{i}=\Phi\left(\mathbf{r}_{i}(t), t\right)$. In ordinary models, the mobility $\chi$ and the diffusion coefficient $D$ are constant. In that case, the statistical equilibrium state is the Boltzmann distribution $\rho \sim e^{-\Phi / T}$ where the temperature $T=1 / \beta$ is given by the Einstein relation $T=D / \chi$. In the present study, we shall consider more general situations and allow the mobility $\chi(\rho)$ and the diffusion coefficient $D(\rho)$ to depend on the local concentration of particles $\rho(\mathbf{r}, t)=\left\langle\sum_{i=1}^{N} \delta\left(\mathbf{r}-\mathbf{r}_{i}(t)\right)\right\rangle$. This is an heuristic approach to take into account microscopic constraints that affect the dynamics of particles at small scales and lead to non-Boltzmannian equilibrium distributions. Indeed, it is not surprising that the mobility or the diffusive properties of a particle depend on its environment. For example, in a dense medium, its motion can be hampered by the presence of the other particles so that its mobility is reduced.

On the other hand, in ordinary models, the particles move in a fixed external potential $\Phi_{\text {ext }}(\mathbf{r})$. In the present study, we want to be more general and take into account the possibility that the potential $\Phi(\mathbf{r}, t)$ is created selfconsistently by the particles themselves. In this paper, we shall neglect statistical correlations and use a mean field description (for more general models taking into account statistical correlations see, e.g., [40,55]). Therefore, we assume that the potential is given by a relation of the form

$$
\Phi(\mathbf{r}, t)=\int \rho\left(\mathbf{r}^{\prime}, t\right) u\left(\left|\mathbf{r}-\mathbf{r}^{\prime}\right|\right) d \mathbf{r}^{\prime}
$$

where $u\left(\left|\mathbf{r}-\mathbf{r}^{\prime}\right|\right)$ is a binary potential of interaction and $\rho(\mathbf{r}, t)$ is the smooth distribution of particles. In general, the mean field approximation gives a very good description of systems with weak long-range binary interactions and it becomes exact in a proper thermodynamic limit $N \rightarrow+\infty$ [40. In Eq. (2), the potential is expressed as a convolution product: $\Phi=u * \rho$. Of course, the potential can be due to the combined effect of the self-interaction and an external field, in which case $\Phi=\Phi_{\text {ext }}+u * \rho$. We shall also consider the case where it is determined by an equation of the form

$$
\epsilon \frac{\partial \Phi}{\partial t}=\Delta \Phi-k^{2} \Phi-\lambda \rho,
$$

where $\epsilon$ and $\lambda$ are positive constants. For $\epsilon=0$, Eq. (3) becomes the screened Poisson equation

$$
\Delta \Phi-k^{2} \Phi=\lambda \rho .
$$

Therefore, we can identify $k^{-1}$ as the screening length. If we assume furthermore that $k=0$, we get the Poisson equation

$$
\Delta \Phi=\lambda \rho .
$$

These last two equations can be put in the form of Eq. (2). Note also that, in the stationary state, Eq. (3) reduces to Eq. (4).

\subsection{Drift-diffusion equations}

For the stochastic process (11), the evolution of the smooth density of particles $\rho(\mathbf{r}, t)$ is governed by the nonlinear mean field Fokker-Planck equation [3340]:

$$
\frac{\partial \rho}{\partial t}=\nabla \cdot[\nabla(D(\rho) \rho)+\chi(\rho) \rho \nabla \Phi]
$$

coupled to Eq. (2) or (3). Let us introduce the notations

$$
D h(\rho)=\frac{d}{d \rho}(\rho D(\rho)), \quad \chi g(\rho)=\rho \chi(\rho),
$$

where $D$ and $\chi$ are positive constants and $h(\rho)$ and $g(\rho)$ are positive functions. These notations are chosen such that the usual stochastic equations with constant diffusion $D(\rho)=D$ and constant mobility $\chi(\rho)=\chi$ are recovered for $h(\rho)=1$ and $g(\rho)=\rho$. With these notations, the nonlinear Fokker-Planck equation (6) can be rewritten

$$
\frac{\partial \rho}{\partial t}=\nabla \cdot(D h(\rho) \nabla \rho+\chi g(\rho) \nabla \Phi) .
$$

It can be put in the conservative form

$$
\frac{\partial \rho}{\partial t}=-\nabla \cdot \mathbf{J}
$$

where

$$
\mathbf{J}=-(D h(\rho) \nabla \rho+\chi g(\rho) \nabla \Phi)
$$

is a diffusion current. This structure guarantees the conservation of mass $M=\int \rho d \mathbf{r}$ provided that the normal component of the current at the boundary vanishes. 


\subsection{Relation to some known models}

Known models can be recovered as particular cases of Eq. (6). When $\Phi_{\text {ext }}$ is an external potential and when $D(\rho)=D$ and $\chi(\rho)=\chi$ are constant, we recover the Smoluchowski equation $\partial_{t} \rho=\nabla \cdot\left(D \nabla \rho+\chi \rho \nabla \Phi_{\text {ext }}\right)$ describing the Brownian motion of colloidal suspensions in a fixed gravitational field 44. When $\rho D(\rho)=K \rho^{\gamma}$ and $\Phi_{\text {ext }}=0$, we recover the porous medium equation $\partial_{t} \rho=$ $K \Delta \rho^{\gamma}$ 22, and when $\Phi_{\text {ext }} \neq 0$, we recover the nonlinear Fokker-Planck equation $\partial_{t} \rho=\nabla \cdot\left(K \nabla \rho^{\gamma}+\chi \rho \nabla \Phi_{\text {ext }}\right)$ introduced by Plastino \& Plastino 21 in connection to the Tsallis statistics 9]. When $D(\rho)=D$ and $\chi(\rho)=\chi$ are constant and $u=-\frac{k}{2 \pi} \cos \left(\theta-\theta^{\prime}\right)$ we obtain the Brownian Mean Field (BMF) model [43, which is the canonical (fixed $T$ ) version of the microcanonical (fixed $E$ ) Hamiltonian Mean Field (HMF) model 44. This is closely related to the Kuramoto model [38] which describes the synchronization of globally coupled nonlinear oscillators. When $D(\rho)=D$ and $\chi(\rho)=\chi$ are constant and $\Phi$ is the gravitational potential satisfying $\Delta \Phi=4 \pi G \rho$, Eqs. (6)(5) become the Smoluchowski-Poisson system describing a model of self-gravitating Brownian particles studied by Chavanis \& Sire 41/42/56/5758/59. When $D(\rho)=D$ and $\chi(\rho)=\chi$ are constant and $\Phi$ is the self-consistent electrostatic potential, we recover the equations introduced by Debye \& Hückel 60] in their model of electrolytes. Models of the form (6)-(4) including a time dependent temperature $\beta(t)$ assuring the conservation of energy have been introduced by Robert \& Sommeria 61. and Chavanis 62. to describe the violent relaxation of two-dimensional vortices in geophysical and astrophysical flows. In the Quasi Geostrophic (QG) model, $k^{-1}$ represents the Rossby length Similar equations have been proposed in [28] to model the violent relaxation of stellar systems. On the other hand, for short range interactions, we can expand the potential in the form $\Phi(\mathbf{r}, t)=a \rho(\mathbf{r}, t)+\frac{b}{2} \Delta \rho(\mathbf{r}, t)$ and Eq. (6) leads to a generalized form of the Cahn-Hilliard equation (see [54/51/55] for details). As a particular case, for $D=0$ and $u=a \delta$ (leading to $\Phi(\mathbf{r}, t)=a \rho(\mathbf{r}, t)$ ), we get $\partial_{t} \rho=\chi a \nabla(g(\rho) \nabla \rho)$ and for $g(\rho)=\rho$, we get the porous medium equation $\partial_{t} \rho=\frac{1}{2} \chi a \Delta \rho^{2}$. Finally, in $d=1$, assuming $D(\rho)=D, \chi(\rho)=\chi$ and $u^{\prime}=-\frac{1}{2 \chi} \delta$, we get the Burgers equation $\partial_{t} \rho+\rho \partial_{x} \rho=D \partial_{x x} \rho$ 63. Therefore, the class of nonlinear mean field Fokker-Planck equations (6)-(2) introduced in 33] can find physical applications in different areas 8 .

\subsection{Generalized Keller-Segel model of chemotaxis}

In addition to the previous examples, nonlinear mean field Fokker-Planck equations can find important applications in the context of chemotaxis 47]. The original Keller-Segel model [49] describing the chemotaxis of bacterial populations consists in two coupled differential equations

$$
\frac{\partial \rho}{\partial t}=\nabla \cdot\left(D_{2} \nabla \rho\right)-\nabla \cdot\left(D_{1} \nabla c\right)
$$

$$
\epsilon \frac{\partial c}{\partial t}=D_{c} \Delta c-k(c) c+f(c) \rho,
$$

that govern the evolution of the density of bacteria $\rho(\mathbf{r}, t)$ and the evolution of the secreted chemical $c(\mathbf{r}, t)$. The bacteria diffuse with a diffusion coefficient $D_{2}$ and they also move in a direction of a positive gradient of the chemical (chemotactic drift). The coefficient $D_{1}$ is a measure of the strength of the influence of the chemical gradient on the flow of bacteria. On the other hand, the chemical is produced by the bacteria with a rate $f(c)$ and is degraded with a rate $k(c)$. It also diffuses with a diffusion coefficient $D_{c}$. In the general Keller-Segel model, $D_{1}=D_{1}(\rho, c)$ and $D_{2}=D_{2}(\rho, c)$ can both depend on the concentration of the bacteria and of the chemical. This takes into account microscopic constraints, like close-packing effects, that can hinder the movement of bacteria. If we assume that the quantities only depend on the concentration of bacteria 4 and write $D_{2}=D h(\rho), D_{1}=\chi g(\rho), k(c)=k^{2}, f(c)=\lambda$ and $D_{c}=1$, we obtain

$$
\begin{gathered}
\frac{\partial \rho}{\partial t}=\nabla \cdot(D h(\rho) \nabla \rho-\chi g(\rho) \nabla c), \\
\epsilon \frac{\partial c}{\partial t}=\Delta c-k^{2} c+\lambda \rho .
\end{gathered}
$$

These equations are isomorphic to the nonlinear mean field Fokker-Planck equations (8)-(3) provided that we make the correspondance $\Phi(\mathbf{r}, t)=-c(\mathbf{r}, t)$ : the potential of interaction is played by the concentration of the secreted chemical.

It is important to note that the Keller-Segel model is a mean field model. If we come back to the exact microscopic equations

$$
\begin{gathered}
\frac{d \mathbf{r}_{i}}{d t}=\chi \nabla c_{d}\left(\mathbf{r}_{i}(t), t\right)+\sqrt{2 D} \mathbf{R}_{i}(t), \\
\epsilon \frac{\partial c_{d}}{\partial t}=\Delta c_{d}-k^{2} c_{d}+\lambda \sum_{i=1}^{N} \delta\left(\mathbf{r}-\mathbf{r}_{i}(t)\right),
\end{gathered}
$$

governing the evolution of each particle (bacteria, cells, social insects, ...), the mean field approximation leading to the standard Keller-Segel model (13)-(14) with $h(\rho)=1$ and $g(\rho)=\rho$ amounts to neglecting statistical correlations; see 6465]66 40/59/67/55] for details. Let us first assume that the system is Markovian and possesses no intrinsic memory in the sense that $c_{d}(\mathbf{r}, t)=-\int u(\mid \mathbf{r}-$ $\left.\mathbf{r}^{\prime} \mid\right) \rho_{d}\left(\mathbf{r}^{\prime}, t\right) d \mathbf{r}^{\prime}$ where $\rho_{d}(\mathbf{r}, t)=\sum_{i=1}^{N} \delta\left(\mathbf{r}-\mathbf{r}_{i}(t)\right)$ is the exact distribution of particles. When the particles interact via a weak long-range binary potential $u\left(\left|\mathbf{r}-\mathbf{r}^{\prime}\right|\right)$, it

\footnotetext{
${ }^{4}$ In this paper, we shall restrict ourselves to this situation. The original Keller-Segel model (11)-(12) where $D_{1}$ and $D_{2}$ depend on both $\rho(\mathbf{r}, t)$ and $c(\mathbf{r}, t)$ does not seem to possess the nice "thermodynamical" properties of the reduced KellerSegel (13)-(14) such as Legendre structure of the free energy functionals, canonical $H$-theorem, Einstein relation etc.
} 
can be shown that the mean field approximation becomes exact in a proper thermodynamic limit $N \rightarrow+\infty$ [40. For example, in Eq. (16) these assumptions correspond to $\epsilon=0$ and $k \ll 1$. By contrast, when memory is not zero and the interaction is short range, corresponding to $\epsilon \neq 0$ and $k \gg 1$ in Eq. (16), the mean field approximation can lead to wrong results. In the context of chemotaxis, the differences between mean field and non mean field models have been discussed by Grima 68 who showed the failure of the mean field approximation for the prediction of the width of the aggregate sizes (the disagreement becomes very severe close to the critical point where we know that mean field approximation breaks down in general). On the other hand, the mean field approximation assumes that the number of particles $N \rightarrow+\infty$. In stellar systems and plasmas, for example, this is always the case. However, for biological systems, the number of interacting bacteria or cells is frequently less than a few thousands so it may be relevant to return to a microscopic description of the bacteria or cells' movement in terms of $N$-body stochastic equations like (15)-(16) as discussed in [67. In this paper, however, we shall exclusively consider mean field models. As we have seen, mean field approximation works well for Markovian systems $(\epsilon=0)$ with weak long-range interactions if (i) $N \rightarrow+\infty$ and (ii) if we are not too close from a critical point. Mean field approximation breaks down: (i) for non-Markovian systems $(\epsilon \neq 0)$ (ii) close to a critical point (iii) for small values of $N$.

\subsection{Generalized free energy and H-theorem}

When $\Phi_{\text {ext }}$ is an external potential, we define the energy by

$$
E=\int \rho \Phi_{e x t} d \mathbf{r}
$$

When $\Phi$ is determined by Eq. (2), the self-interaction energy is given by

$$
E=\frac{1}{2} \int \rho \Phi d \mathbf{r}
$$

Finally, when $\Phi$ is determined by Eq. (3), the energy is

$$
E=\frac{1}{2 \lambda} \int\left[(\nabla \Phi)^{2}+k^{2} \Phi^{2}\right] d \mathbf{r}+\int \rho \Phi d \mathbf{r} .
$$

For $\epsilon=0$, the expression (19) reduces to Eq. (18). On the other hand, we define the temperature by

$$
T=\frac{D}{\chi} .
$$

Therefore, the Einstein relation is preserved in the generalized thermodynamical framework. We also set $\beta=1 / T$. We introduce the generalized entropic functional

$$
S=-\int C(\rho) d \mathbf{r},
$$

where $C(\rho)$ is a convex function $\left(C^{\prime \prime} \geq 0\right)$ defined by

$$
C^{\prime \prime}(\rho)=\frac{h(\rho)}{g(\rho)} .
$$

Note that the relation (22) defines the function $C(\rho)$ up to a term of the form $A \rho+B$. After integration over the domain, the first term is proportional to the mass which is a conserved quantity and the second term is just a constant. Therefore, these terms play no physical role. However, we can adapt the values of the constants $A$ and $B$ in order to obtain convenient expressions of the entropy.

Since the system is dissipative, the energy is not conserved. What is fixed instead of the energy is the temperature defined by the Einstein relation (20). Since $D \propto T$, the temperature measures the strength of the stochastic force in Eq. (10). This corresponds to a canonical description where the system is in contact with a heat bath. Note that the heat bath is completely disconnected from the long-range potential of interaction; it corresponds to short-range interactions modelled by the stochastic term in Eq. (10. For an isolated system described by the microcanonical ensemble the proper thermodynamical potential is the entropy. Alternatively, for a dissipative system described by the canonical ensemble, the relevant thermodynamical potential is the free energy. We introduce the generalized free energy

$$
F=E-T S .
$$

The definition of the free energy (Legendre transform) is preserved in the generalized thermodynamical framework.

When the energy is given by Eqs. (17) or (18), a straightforward calculation (see Appendix G) shows that

$$
\dot{F}=-\int \frac{1}{\chi g(\rho)}(D h(\rho) \nabla \rho+\chi g(\rho) \nabla \Phi)^{2} d \mathbf{r} .
$$

When the energy is given by Eq. (19), we obtain

$$
\begin{array}{r}
\dot{F}=-\frac{1}{\lambda \epsilon} \int\left(\Delta \Phi-k^{2} \Phi-\lambda \rho\right)^{2} d \mathbf{r} \\
-\int \frac{1}{\chi g(\rho)}(D h(\rho) \nabla \rho+\chi g(\rho) \nabla \Phi)^{2} d \mathbf{r} .
\end{array}
$$

Therefore, $\dot{F} \leq 0$. This forms an $H$ theorem in the canonical ensemble. It is sometimes useful to introduce the Massieu function

$$
J=S-\beta E,
$$

which is related to the free energy by $J=-\beta F$. Clearly, we have $\dot{J} \geq 0$. We can now consider particular cases: if $D=0$ (leading to $T=0$ ), we get $F=E$ so that $\dot{E} \leq 0$. If $\chi=0$ (leading to $\beta=0$ ), we have $J=S$ so that $\dot{S} \geq 0$.

\subsection{Stationary solution}

The steady state of Eq. (8) satisfies $\dot{F}=0$ leading to $\mathbf{J}=\mathbf{0}$ or explicitly

$$
D h(\rho) \nabla \rho+\chi g(\rho) \nabla \Phi=\mathbf{0} .
$$


Using Eqs. (20) and (22), we get

$$
C^{\prime \prime}(\rho) \nabla \rho+\beta \nabla \Phi=\mathbf{0},
$$

which can be integrated into

$$
C^{\prime}(\rho)=-\beta \Phi-\alpha
$$

where $\alpha$ is a constant of integration. Since $C$ is convex, this equation can be reversed to give

$$
\rho(\mathbf{r})=F(\beta \Phi(\mathbf{r})+\alpha)
$$

where $F(x)=\left(C^{\prime}\right)^{-1}(-x)$ is a monotonically decreasing function. Thus, in the steady state, the density is a monotonically decreasing function $\rho=\rho(\Phi)$ of the potential. We have the identity

$$
\rho^{\prime}(\Phi)=-\frac{\beta}{C^{\prime \prime}(\rho)} .
$$

Substituting Eq. (2) in Eq. (29), we find that the density profile is determined by an integro-differential equation of the form

$$
C^{\prime}(\rho)=-\beta \int \rho\left(\mathbf{r}^{\prime}\right) u\left(\left|\mathbf{r}-\mathbf{r}^{\prime}\right|\right) d \mathbf{r}^{\prime}-\alpha .
$$

More specifically, when the potential is given by Eq. (41), we obtain a mean field equation of the form

$$
\Delta \Phi-k^{2} \Phi=\lambda F(\beta \Phi+\alpha) .
$$

The constant of integration $\alpha$ is determined by the total mass $M$ (which is a conserved quantity). Finally, we note that the generalized entropy (21) is related to the distribution (30) by 69:

$$
C(\rho)=-\int^{\rho} F^{-1}(x) d x .
$$

Equation (30) determines the distribution from the entropy and Eq. (34) determines the entropy from the distribution.

\subsection{Minimum of free energy}

The critical points of free energy at fixed mass are determined by the variational problem

$$
\delta F+T \alpha \delta M=0,
$$

where $\alpha$ is a Lagrange multiplier. We first consider the case where $\Phi$ is given by Eq. (2). Therefore, the free energy that we consider is

$$
F[\rho]=\frac{1}{2} \int \rho \Phi d \mathbf{r}+T \int C(\rho) d \mathbf{r} .
$$

After straightforward calculations, we find that Eq. 355 leads to

$$
C^{\prime}(\rho)=-\beta \Phi-\alpha
$$

Therefore, comparing with Eq. (29), we find that a stationary solution of Eq. (8) is a critical point of $F$ at fixed mass. Furthermore, it is shown in Appendix B that a steady state of Eq. (8) is linearly dynamically stable iff it is a minimum (at least local) of $F$ at fixed mass. This property also results from Lyapunov's direct method 36. Indeed, we have established that

$$
\dot{F} \leq 0, \quad \dot{F}=0 \leftrightarrow \partial_{t} \rho=0 .
$$

This implies that $\rho(\mathbf{r})$ is linearly dynamically stable iff it is a (local) minimum of $F$ at fixed mass (maxima or saddle points of $F$ are dynamically unstable). In this sense, dynamical and generalized thermodynamical stability in the canonical ensemble coincide. Furthermore, if $F$ is bounded from below 5, we can conclude from Lyapunov's theory that the system will converge to a stable steady state for $t \rightarrow+\infty$ which is a (local) minimum of $F[\rho]$ at fixed mass. If several local minima exist, the choice of the final steady state will depend on a complicated notion of basin of attraction.

In conclusion, a steady solution of the nonlinear mean field Fokker-Planck equation (8) is stable iff it satisfies the minimization problem:

$$
\min _{\rho}\{F[\rho] \quad \mid \quad M[\rho]=M\} .
$$

Taking the second variations of $F$ and using Eq. (31), the condition of stability can be written

$$
\delta^{2} F[\delta \rho]=-\frac{1}{2}\left\{\int \frac{(\delta \rho)^{2}}{\rho^{\prime}(\Phi)} d \mathbf{r}-\int \delta \rho \delta \Phi d \mathbf{r}\right\} \geq 0,
$$

for all perturbations $\delta \rho$ that conserve mass. If $\Phi_{\text {ext }}(\mathbf{r})$ is an external potential, the second term in Eq. (40) vanishes. Therefore, the second variations of the free energy are always positive $\delta^{2} F=-T \delta^{2} S=(1 / 2) T \int C^{\prime \prime}(\rho)(\delta \rho)^{2} \geq 0$ so that a critical point of $F$ at fixed mass is necessarily a minimum. If the potential is given by Eq. (3), the free energy is a functional $F[\rho, \Phi]$ of the density $\rho$ and potential $\Phi$. The cancellation of the first order variations of $F$ with respect to $\delta \rho$ and $\delta \Phi$ yields Eqs. (37) and (4), respectively. The condition of stability can be written

$$
\begin{array}{r}
\delta^{2} F[\delta \rho, \delta \Phi]=-\frac{1}{2} \int \frac{(\delta \rho)^{2}}{\rho^{\prime}(\Phi)} d \mathbf{r}+\int \delta \rho \delta \Phi d \mathbf{r} \\
-\frac{1}{2 \lambda} \int\left(\Delta \delta \Phi-k^{2} \delta \Phi\right) \delta \Phi d \mathbf{r} \geq 0
\end{array}
$$

for all perturbations $\delta \rho$ and $\delta \Phi$ that conserve mass. From now on, we shall only consider the case where the potential is given by Eq. (2) since the case of Eq. (3) can be treated similarly by following the lines sketched above.

\footnotetext{
${ }^{5}$ There are important cases, like the system of selfgravitating Brownian particles, where the free energy is not bounded from below. In that case, the system can either relax towards a local minimum of $F$ at fixed mass (when it exists) or collapse to a Dirac peak [56], leading to a divergence of the free energy $F(t) \rightarrow-\infty$.
} 


\subsection{Particular cases}

If we take $h(\rho)=1$ and $g(\rho)=1 / C^{\prime \prime}(\rho)$, we get

$$
\frac{\partial \rho}{\partial t}=\nabla \cdot\left(D \nabla \rho+\frac{\chi}{C^{\prime \prime}(\rho)} \nabla \Phi\right) .
$$

In that case, we have a constant diffusion $D(\rho)=D$ and a variable mobility $\chi(\rho)=\chi /\left(\rho C^{\prime \prime}(\rho)\right)$. If we take $g(\rho)=\rho$ and $h(\rho)=\rho C^{\prime \prime}(\rho)$, we get

$$
\frac{\partial \rho}{\partial t}=\nabla \cdot\left(D \rho C^{\prime \prime}(\rho) \nabla \rho+\chi \rho \nabla \Phi\right) .
$$

In that case, we have a constant mobility $\chi(\rho)=\chi$ and a variable diffusion $D(\rho)=D \rho[C(\rho) / \rho]^{\prime}$. Note that the condition $D(\rho) \geq 0$ requires that $[C(\rho) / \rho]^{\prime} \geq 0$. This gives a constraint on the possible forms of $C(\rho)$.

\subsection{Generalized Smoluchowski equation}

The NFP equation (43) can be put in the form of a generalized Smoluchowski (GS) equation

$$
\frac{\partial \rho}{\partial t}=\nabla \cdot[\chi(\nabla p+\rho \nabla \Phi)],
$$

with a barotropic equation of state $p(\rho)$ given by

$$
p^{\prime}(\rho)=T \rho C^{\prime \prime}(\rho) \text {. }
$$

Since $C$ is convex, we have $p^{\prime}(\rho) \geq 0$. On the other hand, integrating Eq. (45) twice, we get

$$
T C(\rho)=\rho \int^{\rho} \frac{p\left(\rho^{\prime}\right)}{\rho^{\prime 2}} d \rho^{\prime} .
$$

Therefore, the free energy (36) can be rewritten

$$
F[\rho]=\frac{1}{2} \int \rho \Phi d \mathbf{r}+\int \rho \int^{\rho} \frac{p\left(\rho^{\prime}\right)}{\rho^{\prime 2}} d \rho^{\prime} d \mathbf{r} .
$$

With these notations, the $H$-theorem (24) becomes

$$
\dot{F}=-\int \frac{\chi}{\rho}(\nabla p+\rho \nabla \Phi)^{2} d \mathbf{r} \leq 0 .
$$

The stationary solutions of the GS equation (44) satisfy the relation

$$
\nabla p+\rho \nabla \Phi=\mathbf{0}
$$

which is similar to a condition of hydrostatic equilibrium. Since $p=p(\rho)$, this relation can be integrated to give $\rho=\rho(\Phi)$ through

$$
\int^{\rho} \frac{p^{\prime}\left(\rho^{\prime}\right)}{\rho^{\prime}} d \rho^{\prime}=-\Phi .
$$

This is equivalent to

$$
\frac{p^{\prime}(\rho)}{\rho}=-\frac{1}{\rho^{\prime}(\Phi)} .
$$

This relation can also be obtained from Eqs. (45) and (31). Therefore, we recover the fact that, in the steady state, $\rho=\rho(\Phi)$ is a monotonically decreasing function of $\Phi$. We also note the identity

$$
p(\rho)=\frac{1}{\chi} D(\rho) \rho=T \rho^{2}\left[\frac{C(\rho)}{\rho}\right]^{\prime}=T\left[C^{\prime}(\rho) \rho-C(\rho)\right] .
$$

Finally, we note that the relation (50), equivalent to the condition of hydrostatic equilibrium (49), can also be obtained by extremizing the free energy (47) at fixed mass writing $\delta F-\alpha \delta M=0$. More precisely, we have the important result: a steady solution of the generalized Smoluchowski equation (44) is linearly dynamically stable iff it is a (local) minimum of the free energy $F[\rho]$ at fixed mass $M[\rho]=M$. This corresponds to the minimization problem (39) with Eq. (47). Note that the GS equation has been introduced here in a very general context. In this sense, it is valid for arbitrary value of $\chi$ (not necessarily small). Alternatively, we shall see in Sec. 4.8 that a generalized Smoluchowski equation with $\chi=1 / \xi \rightarrow 0$ can be derived from a generalized Kramers equation in the strong friction limit $\xi \rightarrow+\infty$.

\subsection{Phenomenological derivation of the nonlinear Fokker-Planck equation}

The form of the diffusion current appearing in the NFP equation (8) can be obtained by different phenomenological procedures.

\subsubsection{Functional derivative}

For a given free energy functional $F[\rho]$, we can introduce phenomenologically a dynamical model by writing the evolution of the density as a continuity equation $\partial_{t} \rho=-\nabla \cdot \mathbf{J}$ where the current is proportional to the gradient of the functional derivative of the free energy [36], i.e.

$$
\frac{\partial \rho}{\partial t}=\nabla \cdot\left[\chi(\mathbf{r}, t) \rho \nabla \frac{\delta F}{\delta \rho}\right] .
$$

For the free energy (36), we have

$$
\frac{\delta F}{\delta \rho}=T C^{\prime}(\rho)+\Phi
$$

so that

$$
\frac{\partial \rho}{\partial t}=\nabla \cdot\left[\chi(\mathbf{r}, t)\left(T \rho C^{\prime \prime}(\rho) \nabla \rho+\rho \nabla \Phi\right)\right] .
$$

This equation is more general than Eq. (8). It shows that, for a given free energy, we can introduce an infinite class of NFP equations where $\chi(\mathbf{r}, t)$ is an arbitrary positive function of position and time. In particular, $\chi(\mathbf{r}, t)$ can be a positive function of the density $\rho(\mathbf{r}, t)$. If we set $\chi(\mathbf{r}, t)=$ 
$\chi g(\rho) / \rho$ we recover Eq. (8) as a particular case. We can also write Eq. (55) in the alternative form

$$
\frac{\partial \rho}{\partial t}=\nabla \cdot\left[\tilde{\chi}(\mathbf{r}, t)\left(T \nabla \rho+\frac{1}{C^{\prime \prime}(\rho)} \nabla \Phi\right)\right],
$$

where $\tilde{\chi}(\mathbf{r}, t)$ is an arbitrary positive function of position and time. If we set $\tilde{\chi}(\mathbf{r}, t)=\chi h(\rho)$ we recover Eq. (8) . These two alternative forms (55) and (56) were given in 33 .

On the other hand, the formulation (53) ensures that the free energy decreases monotonically provided that $\chi(\mathbf{r}, t)$ is positive. Indeed,

$$
\begin{array}{r}
\dot{F}=\int \frac{\delta F}{\delta \rho} \frac{\partial \rho}{\partial t} d \mathbf{r}=-\int \frac{\delta F}{\delta \rho} \nabla \cdot \mathbf{J} d \mathbf{r} \\
=\int \mathbf{J} \cdot \nabla \frac{\delta F}{\delta \rho} d \mathbf{r}=-\int \chi(\mathbf{r}, t) \rho\left(\nabla \frac{\delta F}{\delta \rho}\right)^{2} d \mathbf{r} \leq 0 .
\end{array}
$$

Furthermore, a steady state of Eq. (53) satisfies $\dot{F}=0$, i.e $\nabla(\delta F / \delta \rho)=0$ leading to

$$
\frac{\delta F}{\delta \rho}=-T \alpha,
$$

where $\alpha$ is a constant of integration. This is equivalent to the extremization of $F$ at fixed mass $M$ if we write the first variations as $\delta F+T \alpha \delta M=0$. Therefore, a steady state extremizes the free energy at fixed mass. Using Eq. (54), we find that the equilibrium density profile satisfies

$$
C^{\prime}(\rho)=-\beta \Phi-\alpha .
$$

Finally, using Lyapunov's direct method, one can show that a steady state $\rho(\mathbf{r})$ of Eq. (53) is linearly dynamically stable iff it is a (local) minimum of $F$ at fixed mass. We again emphasize that the stationary states of Eq. (55), and the $H$-theorem, only depend on the form of the free energy $F[\rho]$ and are independent on the positive function $\chi(\mathbf{r}, t)$. These properties are therefore valid for the whole class of NFP equations associated with a given free energy functional. Therefore, for a given free energy specified by $C(\rho)$, we can construct an infinite class of NFP equations with arbitrary $\chi(\mathbf{r}, t)$ that have the same equilibrium states (59) but a different dynamics.

\subsubsection{Onsager's linear thermodynamics}

The previous approach is equivalent to Onsager's linear thermodynamics. Indeed, at equilibrium, we expect that the distribution $\rho(\mathbf{r})$ minimizes the free energy $F$ at fixed mass. This leads to Eq. (58) or (59). Noting that the chemical potential

$$
\lambda(\mathbf{r}, t) \equiv \frac{\delta F}{\delta \rho}=T C^{\prime}(\rho)+\Phi,
$$

is uniform at equilibrium, the linear thermodynamics of Onsager suggests that, close to equilibrium, the current is proportional to the gradient of the chemical potential. If we write

$$
\mathbf{J}=-\chi(\mathbf{r}, t) \rho \nabla \lambda(\mathbf{r}, t),
$$

the linear thermodynamics of Onsager leads to Eq. (53).

\subsubsection{Maximum Free Energy Dissipation Principle}

The same results can be obtained from a variational formulation, called the Maximum Free Energy Dissipation (MFED) principle which is the canonical ensemble version of the Maximum Entropy Production (MEP) principle [33. At equilibrium, the optimal distribution $\rho(\mathbf{r})$ minimizes the free energy $F[\rho]$ at fixed mass $M$. Out of equilibrium, we may expect that the optimal current $\mathbf{J}$ maximizes the rate of free energy dissipation $\dot{F}[\mathbf{J}]$ under some constraints. This can be viewed as a variational formulation of Onsager's linear thermodynamics. The rate of dissipation of free energy is given by

$$
\dot{F}=\int \frac{\delta F}{\delta \rho} \frac{\partial \rho}{\partial t} d \mathbf{r}=-\int \frac{\delta F}{\delta \rho} \nabla \cdot \mathbf{J} d \mathbf{r}=\int \mathbf{J} \cdot \nabla \frac{\delta F}{\delta \rho} d \mathbf{r} .
$$

We shall determine the optimal current $\mathbf{J}_{*}$ which maximizes the rate of dissipation of free energy $\dot{F}$ under the constraint $J^{2} \leq C(\mathbf{r}, t)$ putting a physical bound on $|\mathbf{J}|$. It can be shown that the bound is always reached so that we can replace the inequality by an equality. Thus, we write the variational problem as

$$
\delta \dot{F}+\delta\left(\int \frac{\mathbf{J}^{2}}{2 \rho \chi(\mathbf{r}, t)} d \mathbf{r}\right)=0,
$$

where $\chi(\mathbf{r}, t)$ is a local Lagrange multiplier. Performing the variations on $\mathbf{J}$, we obtain

$$
\mathbf{J}_{*}=-\chi(\mathbf{r}, t) \rho \nabla \frac{\delta F}{\delta \rho},
$$

which returns Eq. (53). Note that if we introduce the "dissipation" function

$$
E_{d} \equiv \int \frac{\mathbf{J}^{2}}{2 \rho \chi(\mathbf{r}, t)} d \mathbf{r}
$$

we have

$$
E_{d}\left[\mathbf{J}_{*}\right]=-\frac{1}{2} \dot{F}\left[\mathbf{J}_{*}\right]
$$

On the other hand, since $\delta^{2}\left(\dot{F}+E_{d}\right)=-\int \frac{(\delta J)^{2}}{2 \rho \chi} d \mathbf{r} \leq 0$, the optimal current (64) maximizes the dissipation of free energy under the constraint $J^{2} \leq C(\mathbf{r}, t)$.

\subsection{Kinetic derivation of the nonlinear Fokker-Planck equation}

Nonlinear Fokker-Planck equation where the diffusion coefficient and the mobility explicitly depend on the local concentration of particles can be derived from a kinetic theory, starting from the master equation, and assuming that the probabilities of transition explicitly depend on the occupation numbers (concentrations) of the initial and arrival states. We briefly summarize the approach developed 
by Kaniadakis 12 and make the link with the phenomenological equations studied previously.

We introduce a stochastic dynamics by defining the probability of transition of a particle from position $\mathbf{r}$ to position $\mathbf{r}^{\prime}$. Following Kaniadakis [12, we assume the following form

$$
\pi\left(\mathbf{r} \rightarrow \mathbf{r}^{\prime}\right)=w\left(\mathbf{r}, \mathbf{r}-\mathbf{r}^{\prime}\right) a[\rho(\mathbf{r}, t)] b\left[\rho\left(\mathbf{r}^{\prime}, t\right)\right] .
$$

Usual stochastic processes correspond to $a(\rho)=\rho$ and $b(\rho)=1$ : the probability of transition is proportional to the density of the initial state and independent on the density of the final state. They lead to the ordinary FokkerPlanck equation (86) as will be shown below. Here, we assume a more general dependence on the occupancy in the initial and arrival states. This can account for microscopic constraints like close-packing effects that can inhibitate the transition. Quite generally, the evolution of the density satisfies the master equation

$$
\frac{\partial \rho}{\partial t}=\int\left[\pi\left(\mathbf{r}^{\prime} \rightarrow \mathbf{r}\right)-\pi\left(\mathbf{r} \rightarrow \mathbf{r}^{\prime}\right)\right] d \mathbf{r}^{\prime} .
$$

Assuming that the evolution is sufficiently slow, and local, such that the dynamics only permits values of $\mathbf{r}^{\prime}$ close to $\mathbf{r}$, one can develop the term in brackets in Eq. (68) in powers of $\mathbf{r}-\mathbf{r}^{\prime}$. Proceeding along the lines of [12, we obtain a Fokker-Planck-like equation

$$
\frac{\partial \rho}{\partial t}=\frac{\partial}{\partial x_{i}}\left[\left(\zeta_{i}+\frac{\partial \zeta_{i j}}{\partial x_{j}}\right) \gamma(\rho)+\gamma(\rho) \frac{\partial \ln \kappa(\rho)}{\partial \rho} \zeta_{i j} \frac{\partial \rho}{\partial x_{j}}\right],
$$

with

$$
\gamma(\rho)=a(\rho) b(\rho), \quad \kappa(\rho)=\frac{a(\rho)}{b(\rho)},
$$

and

$$
\begin{aligned}
\zeta_{i}(\mathbf{r}) & =-\int y_{i} w(\mathbf{r}, \mathbf{y}) d \mathbf{y}, \\
\zeta_{i j}(\mathbf{r}) & =\frac{1}{2} \int y_{i} y_{j} w(\mathbf{r}, \mathbf{y}) d \mathbf{y} .
\end{aligned}
$$

The moments $\zeta_{i}$ and $\zeta_{i j}$ are fixed by the ordinary Langevin equation

$$
\frac{d \mathbf{r}}{d t}=-\chi \nabla \Phi+\sqrt{2 D} \mathbf{R}(t),
$$

where $\chi$ and $D$ are constant. Assuming isotropy $\zeta_{i}=J_{i}$, $\zeta_{i j}=D \delta_{i j}$, the kinetic equation (69) becomes

$$
\frac{\partial \rho}{\partial t}=\nabla \cdot\left[(\mathbf{J}+\nabla D) \gamma(\rho)+\gamma(\rho) \frac{\partial \ln \kappa(\rho)}{\partial \rho} D \nabla \rho\right] .
$$

Now, according to the Langevin equation (73), $D$ is independent on $\mathbf{r}$ and $\mathbf{J}=\chi \nabla \Phi$. Thus, we get

$$
\frac{\partial \rho}{\partial t}=\nabla \cdot\left[D \gamma(\rho) \frac{\partial \ln \kappa(\rho)}{\partial \rho} \nabla \rho+\chi \gamma(\rho) \nabla \Phi\right] .
$$

If we define

$$
h(\rho)=\gamma(\rho) \frac{\partial \ln \kappa(\rho)}{\partial \rho}, \quad g(\rho)=\gamma(\rho),
$$

the foregoing equation can be written

$$
\frac{\partial \rho}{\partial t}=\nabla \cdot[D h(\rho) \nabla \rho+\chi g(\rho) \nabla \Phi],
$$

and it coincides 6 with the phenomenological equation (8). We note that

$$
\ln \kappa(\rho)=C^{\prime}(\rho) .
$$

We also have the relations

$$
\begin{gathered}
a(\rho)=\sqrt{\gamma(\rho) \kappa(\rho)}=\sqrt{g(\rho)} e^{C^{\prime}(\rho) / 2} \\
b(\rho)=\sqrt{\frac{\gamma(\rho)}{\kappa(\rho)}}=\sqrt{g(\rho)} e^{-C^{\prime}(\rho) / 2}
\end{gathered}
$$

Inversely

$$
\begin{gathered}
g(\rho)=a(\rho) b(\rho), \quad C^{\prime}(\rho)=\ln \left[\frac{a(\rho)}{b(\rho)}\right], \\
h(\rho)=b(\rho) a^{\prime}(\rho)-a(\rho) b^{\prime}(\rho) .
\end{gathered}
$$

It seems natural to assume that the transition probability is proportional to the density of the initial state so that $a(\rho)=\rho$. In that case, we obtain an equation of the form

$$
\frac{\partial \rho}{\partial t}=\nabla \cdot\left(D\left[b(\rho)-\rho b^{\prime}(\rho)\right] \nabla \rho+\chi \rho b(\rho) \nabla \Phi\right) .
$$

Note that the coefficients of diffusion and mobility are not independent since they are both expressed in terms of $b(\rho)$. Choosing $b(\rho)=1$, i.e. a probability of transition which does not depend on the population of the arrival state, leads to the standard Fokker-Planck equation (86). If, now, we assume that the transition probability is blocked (inhibited) if the concentration of the arrival state is equal to $\sigma_{0}$, then it seems natural to take $b(\rho)=1-\rho / \sigma_{0}$. In that case, we obtain

$$
\frac{\partial \rho}{\partial t}=\nabla \cdot\left(D \nabla \rho+\chi \rho\left(1-\rho / \sigma_{0}\right) \nabla \Phi\right),
$$

which will be considered in Sec. 3.5 Inversely, we can wonder what the general form of the mobility will be if we assume a normal diffusion $h(\rho)=1$. This leads to $b(\rho)-\rho b^{\prime}(\rho)=1$ which is integrated in $b(\rho)=1+K \rho$ where $K$ is a constant. Interestingly, we find that this condition selects the class of fermions $(K=-1)$ and bosons $(K=+1)$ and intermediate statistics (arbitrary $K)$. The corresponding NFP equation is

$$
\frac{\partial \rho}{\partial t}=\nabla \cdot(D \nabla \rho+\chi \rho(1+K \rho) \nabla \Phi),
$$

which will be considered in Sec. 3.6.

\footnotetext{
${ }^{6}$ In Sec. 2.1 we have obtained generalized Fokker-Planck equations by using ordinary Master equations (based on usual transition probabilities $a(\rho)=\rho$ and $b(\rho)=1$ ) and generalized Langevin equations where the diffusion coefficient and the mobility depend on the density. In this section, we have obtained generalized Fokker-Planck equations by using generalized Master equations (based on density dependent transition probabilities) and ordinary Langevin equations with constant coefficients.
} 


\section{Examples of nonlinear Smoluchowski equations and generalized Keller-Segel models}

In this section, we give several explicit examples of nonlinear mean field Fokker-Planck equations. Some correspond to well-known forms of entropies, and others are new. We emphasize that these equations can have applications in different domains of physics as discussed in Sec. 2.3. Importantly, they can provide generalized Keller-Segel models of chemotaxis (see Sec. 2.4). Most of these models have not been considered before in biology because the connection with generalized thermodynamics was not made. This is why we give a relatively detailed description of these models since their applications in biology are new.

\subsection{Standard model: Boltzmann entropy}

If we take $h(\rho)=1$ and $g(\rho)=\rho$, we get the ordinary Smoluchowski equation

$$
\frac{\partial \rho}{\partial t}=\nabla \cdot(D \nabla \rho+\chi \rho \nabla \Phi) .
$$

It corresponds to an ordinary diffusion $D(\rho)=D$ and a constant mobility $\chi(\rho)=\chi$. The associated stochastic process is

$$
\frac{d \mathbf{r}}{d t}=-\chi \nabla \Phi+\sqrt{2 D} \mathbf{R}(t)
$$

The entropy is the Boltzmann entropy

$$
S=-\int \rho \ln \rho d \mathbf{r}
$$

and the stationary solution of Eq. (86) is the Boltzmann distribution

$$
\rho=e^{-\beta \Phi-\alpha-1}
$$

The pressure law is

$$
p(\rho)=\rho T \text {. }
$$

This is similar to the equation of state for an isothermal gas with constant temperature $T$. When the FokkerPlanck equation (86) is coupled to the Poisson equation (5), we obtain the Smoluchowski-Poisson system describing a gas of self-gravitating Brownian particles 414256575859 . When the Fokker-Planck equation (86) is coupled to the field Eq. (3), we obtain the standard Keller-Segel model describing the chemotactic aggregation of biological populations 52.

\subsection{Power law diffusion: Tsallis entropy}

If we take $h(\rho)=q \rho^{q-1}$ and $g(\rho)=\rho$, we obtain the NFP equation

$$
\frac{\partial \rho}{\partial t}=\nabla \cdot\left(D \nabla \rho^{q}+\chi \rho \nabla \Phi\right)
$$

It corresponds to a power law diffusion $D(\rho)=D \rho^{q-1}$ and a constant mobility $\chi(\rho)=\chi$. This equation was introduced by Plastino \& Plastino [21]. The associated stochastic process, introduced by Borland 25, is

$$
\frac{d \mathbf{r}}{d t}=-\chi \nabla \Phi+\sqrt{2 D} \rho^{\frac{q-1}{2}} \mathbf{R}(t)
$$

This model can take into account effects of non-ergodicity and nonextensivity. It leads to a situation of anomalous diffusion related to the Tsallis statistics. For $q=1$, we recover the standard Brownian model with a constant diffusion coefficient, corresponding to a pure random walk. In that case, the sizes of the random kicks are uniform and do not depend on where the particle happens to be. For $q \neq 1$, the size of the random kicks changes, depending on the distribution of the particles around the "test" particle. A particle which is in a region that is highly populated [large $\rho(\mathbf{r}, t)$ ] will tend to have larger kicks if $q>1$ and smaller kicks if $q<1$. Since the microscopics depends on the actual density in space, this creates a bias in the ergodic behavior of the system. Then, the dynamics has a fractal or multi-fractal phase space structure [25. The generalized entropy associated to Eq. (91) is the Tsallis entropy

$$
S=-\frac{1}{q-1} \int\left(\rho^{q}-\rho\right) d \mathbf{r}
$$

and the stationary solution is the Tsallis distribution

$$
\rho=\left(\frac{1}{q}\right)^{\frac{1}{q-1}}[1-(q-1)(\beta \Phi+\alpha)]_{+}^{1 /(q-1)} .
$$

The pressure law is

$$
p(\rho)=T \rho^{q}
$$

This is similar to a polytropic gas with an equation of state $p=K \rho^{\gamma}$ where $K=T$ plays the role of a polytropic temperature and $q=\gamma$ is the polytropic index (we also set $\gamma=1+1 / n)$. Note that the Tsallis entropy can be written

$$
S=-\int \rho \ln _{(q)} \rho d \mathbf{r}
$$

$$
\ln _{(q)}(x)=\frac{1}{q-1}\left(x^{q-1}-1\right) .
$$

The stationary solution can be written

$$
\rho=\left(\frac{1}{q}\right)^{\frac{1}{q-1}} e_{(q)}^{-\beta \Phi-\alpha}
$$

with the $q$-exponential

$$
e_{(q)}(x)=[1+(q-1) x]^{\frac{1}{q-1}} .
$$


For $q=1$, we recover the standard model (86). For $q=$ 2 , we have some simplifications. In that case, the NFP equation (91) becomes

$$
\frac{\partial \rho}{\partial t}=\nabla \cdot\left(D \nabla \rho^{2}+\chi \rho \nabla \Phi\right)
$$

The entropy is the quadratic functional

$$
S=-\int \rho^{2} d \mathbf{r},
$$

and the stationary solution is

$$
\rho=-\frac{1}{2}(\beta \Phi+\alpha),
$$

corresponding to a linear relation between the density and the potential. In that case, the differential equation (33) determining the steady state reduces to the Helmholtz equation. Finally, the pressure is

$$
p(\rho)=T \rho^{2},
$$

corresponding to a polytrope with index $n=1$. When the NFP equation (91) is coupled to the Poisson equation (5), we obtain the polytropic Smoluchowski-Poisson system describing self-gravitating Langevin particles. When the NFP equation (91) is coupled to the field Eq. (3), we obtain a generalized Keller-Segel model of chemotaxis taking into account anomalous diffusion. These models have been introduced by Chavanis \& Sire [4515153.

\subsection{Logotropic distributions: log-entropy}

If we take $h(\rho)=1 / \rho$ and $g(\rho)=\rho$, we obtain

$$
\frac{\partial \rho}{\partial t}=\nabla \cdot(D \nabla \ln \rho+\chi \rho \nabla \Phi) .
$$

The generalized entropy associated to Eq. (104) is the logentropy

$$
S=\int \ln \rho d \mathbf{r}
$$

and the stationary solution is

$$
\rho=\frac{1}{\alpha+\beta \Phi} .
$$

For a quadratic potential $\Phi_{\text {ext }}=r^{2} / 2$, this corresponds to the Lorentzian function. The pressure is

$$
p(\rho)=T \ln \rho .
$$

This is similar to a logotropic equation of state [70]. This is also connected to a polytropic equation of state (or Tsallis distribution) with $\gamma=q=0$. Indeed, the logotropic model (104) can be deduced from Eq. (91) by writing $D \nabla \rho^{q}=$ $D q \rho^{q-1} \nabla \rho$, taking $q=0$ and re-defining $D q \rightarrow D$. When the NFP equation (104) is coupled to the Poisson equation (5), we obtain the logotropic Smoluchowski-Poisson system. When the NFP equation (104) is coupled to the field Eq. (3), we obtain a generalized Keller-Segel model of chemotaxis. These models have been introduced by Chavanis \& Sire 71 .

\subsection{Power law diffusion and drift: Tsallis entropy}

We introduce here a new model generalizing the polytropic model (91). If we take $h(\rho)=q \rho^{q+\mu-1}$ and $g(\rho)=\rho^{\mu+1}$, we obtain

$$
\frac{\partial \rho}{\partial t}=\nabla \cdot\left(D q \rho^{q+\mu-1} \nabla \rho+\chi \rho^{\mu+1} \nabla \Phi\right) .
$$

This corresponds to a power law diffusion $D(\rho)=\frac{D q}{q+\mu} \rho^{q+\mu-1}$ and a power law mobility $\chi(\rho)=\chi \rho^{\mu}$. The associated stochastic process is

$$
\frac{d \mathbf{r}}{d t}=-\chi \rho^{\mu} \nabla \Phi+\sqrt{\frac{2 D q}{q+\mu}} \rho^{\frac{q+\mu-1}{2}} \mathbf{R}(t) .
$$

Since $\rho^{\mu}$ can be put in factor of the diffusion current in Eq. (108), this model belongs to the infinite family of NFP equations associated to the Tsallis entropy with index $q$ (see discussion in Sec. 2.10.1).

For $\mu=0$, we recover Eq. (91) with a constant mobility and a power law diffusion. For $(\mu, q)=(0,0)$, we recover the logotropic Smoluchowski equation (104) provided that we make the transformation $D q \rightarrow D$. For $\mu=1-q$, we have a normal diffusion and a power law mobility

$$
\frac{\partial \rho}{\partial t}=\nabla \cdot\left(D q \nabla \rho+\chi \rho^{2-q} \nabla \Phi\right) .
$$

For $q=2$, we get

$$
\frac{\partial \rho}{\partial t}=\nabla \cdot(2 D \nabla \rho+\chi \nabla \Phi)
$$

which has the same equilibrium states as Eq. (100). If we assume furthermore that $\Phi$ is given by the Poisson equation (5), Eq. (111) reduces to the linear equation

$$
\frac{\partial \rho}{\partial t}=2 D \Delta \rho+\chi \lambda \rho .
$$

Finally, for $q=0$ (making the transformation $q D \rightarrow D$ ), we obtain

$$
\frac{\partial \rho}{\partial t}=\nabla \cdot\left(D \nabla \rho+\chi \rho^{2} \nabla \Phi\right)
$$

which has the same equilibrium states as Eq. (104). When the NFP equation (108) is coupled to the field equation (3), we obtain a generalized Keller-Segel model of chemotaxis taking into account anomalous diffusion and anomalous mobility. This model will be studied in a forthcoming paper, in continuity with 45 .

\subsection{Filling factor: Fermi-Dirac entropy}

If we take $h(\rho)=1$ and $g(\rho)=\rho\left(1-\rho / \sigma_{0}\right)$, we obtain

$$
\frac{\partial \rho}{\partial t}=\nabla \cdot\left(D \nabla \rho+\chi \rho\left(1-\rho / \sigma_{0}\right) \nabla \Phi\right) .
$$


This corresponds to a normal diffusion $D(\rho)=D$ and a mobility $\chi(\rho)=\chi\left(1-\rho / \sigma_{0}\right)$ vanishing linearly when the density reaches the maximum value $\rho_{\max }=\sigma_{0}$. The associated stochastic process is

$$
\frac{d \mathbf{r}}{d t}=-\chi\left(1-\rho / \sigma_{0}\right) \nabla \Phi+\sqrt{2 D} \mathbf{R}(t) .
$$

The generalized entropy associated with Eq. (114) is the Fermi-Dirac entropy in position space

$$
S=-\sigma_{0} \int\left\{\frac{\rho}{\sigma_{0}} \ln \frac{\rho}{\sigma_{0}}+\left(1-\frac{\rho}{\sigma_{0}}\right) \ln \left(1-\frac{\rho}{\sigma_{0}}\right)\right\} d \mathbf{r}
$$

and the stationary solution is the Fermi-Dirac distribution in position space

$$
\rho=\frac{\sigma_{0}}{1+e^{\beta \Phi+\alpha}} .
$$

From Eq. (117), we see that, in the stationary state, $\rho \leq$ $\sigma_{0}$. This bound is similar to the Pauli exclusion principle in quantum mechanics. In fact, we can show that $\rho(\mathbf{r}, t)$ remains bounded by $\sigma_{0}$ during the whole evolution. For $\sigma_{0} \rightarrow+\infty$, we recover the standard model 866).

An alternative model, with the same entropy and the same equilibrium states, is obtained by taking $h(\rho)=$ $1 /\left(1-\rho / \sigma_{0}\right)$ and $g(\rho)=\rho$. This leads to

$$
\frac{\partial \rho}{\partial t}=\nabla \cdot\left(-D \sigma_{0} \nabla \ln \left(1-\rho / \sigma_{0}\right)+\chi \rho \nabla \Phi\right) .
$$

This corresponds to a nonlinear diffusion with $D(\rho)=$ $-\sigma_{0}(D / \rho) \ln \left(1-\rho / \sigma_{0}\right)$ and a constant mobility $\chi(\rho)=\chi$. Equation (118) can be put in the form of a generalized Smoluchowski equation (44) with a pressure law

$$
p(\rho)=-T \sigma_{0} \ln \left(1-\rho / \sigma_{0}\right) .
$$

For $\rho \ll \sigma_{0}$, we recover the "isothermal" equation of state $p=\rho T$ leading to the standard model (86). However, for higher densities, the equation of state is modified and the pressure diverges when $\rho \rightarrow \sigma_{0}$. This prevents the density from exceeding the maximum value $\sigma_{0}$.

In the context of chemotaxis, the model (114) has been introduced by Hillen \& Painter 72$]$ and, independently, by Chavanis 3366733. It provides a regularization of the standard Keller-Segel model preventing overcrowding, blow-up and unphysical singularities. The filling factor $\left(1-\rho / \sigma_{0}\right)$ takes into account the fact that the particles cannot interpenetrate because of their finite size $a$. Therefore, the maximum allowable density is $\sigma_{0} \sim 1 / a^{d}$. It is achieved when all the cells are packed together. In the model (114), it is assumed that the mobility vanishes when the density reaches the close packing value $\left(\rho \rightarrow \sigma_{0}\right)$ while the diffusion is not affected. The alternative model (118) has been introduced in Chavanis 33173 . In that case, the mobility is assumed to be constant and the regularization preventing overcrowding is taken into account in the pressure law (119). As explained in Sec. 2.10.1, we can multiply the diffusion term and the mobility term by the same positive function $\chi(\mathbf{r}, t)$ in order to obtain a more general model with the same entropy and the same equilibrium states. Note finally that an equation similar to Eq. (114) has been introduced by Robert \& Sommeria 61 (see also [28]) in the statistical mechanics of two-dimensional turbulence for two vorticity levels 0 and $\sigma_{0}$. In that case, $\rho$ represents the coarse-grained vorticity $\bar{\omega}$ and $\Phi$ plays the role of the stream function $\psi$. The "exclusion principle" leading to the Fermi-Dirac entropy (116) is a consequence of the 2D Euler equation implying that the vorticity levels cannot overlap so that $\bar{\omega}(\mathbf{r}, t) \leq \sigma_{0}$. These analogies between chemotaxis and $2 \mathrm{D}$ turbulence are further discussed in [73].

\subsection{Fermi, Bose and intermediate statistics}

If we take $h(\rho)=1$ and $g(\rho)=\rho(1+K \rho)$, we obtain

$$
\frac{\partial \rho}{\partial t}=\nabla \cdot(D \nabla \rho+\chi \rho(1+K \rho) \nabla \Phi) .
$$

This corresponds to a normal diffusion $D(\rho)=D$ and a variable mobility $\chi(\rho)=\chi(1+K \rho)$. The associated stochastic process is

$$
\frac{d \mathbf{r}}{d t}=-\chi(1+K \rho) \nabla \Phi+\sqrt{2 D} \mathbf{R}(t) .
$$

Here, $K$ is a real number taking positive or negative values. When $K>0$ the mobility is enhanced in regions of large densities and when $K<0$, it is reduced. This takes into account inclusion $(K>0)$ or exclusion $(K<0)$ principles. For $K=0$, we recover the standard model (866). The generalized entropy associated with Eq. (120) is

$$
S=-\int\left[\rho \ln \rho-\frac{1}{K}(1+K \rho) \ln (1+K \rho)\right] d \mathbf{r},
$$

and the stationary solution is

$$
\rho=\frac{1}{e^{\beta \Phi+\alpha}-K} .
$$

For $K=+1$ we obtain the Bose-Einstein statistics and for $K=-1$ we obtain the Fermi-Dirac statistics. For other values of $K$, we obtain intermediate statistics (quons) interpolating between fermions and bosons. For $K=0$, we recover the Boltzmann statistics.

An alternative model with the same entropy and the same equilibrium states is obtained by taking $h(\rho)=1 /(1+$ $K \rho)$ and $g(\rho)=\rho$. This leads to

$$
\frac{\partial \rho}{\partial t}=\nabla \cdot\left(\frac{1}{K} \nabla \ln (1+K \rho)+\chi \rho \nabla \Phi\right) .
$$

This corresponds to a nonlinear diffusion such that $D(\rho)=$ $(D / K \rho) \ln (1+K \rho)$ and a constant mobility $\chi(\rho)=\chi$. The pressure law is

$$
p(\rho)=\frac{T}{K} \ln (1+K \rho) .
$$


We recall that Eq. 120) can be obtained from the master equation (68) when the transition probabilities are of the form (67) with $a(\rho)=\rho$ and $b(\rho)=1+K \rho$. Alternatively, the model (124) corresponds to $a(\rho)=\rho / \sqrt{1+K \rho}$ and $b(\rho)=\sqrt{1+K \rho}$.

The NFP equations (120) and (124) have been introduced by Kaniadakis \& Quarati 27] (see also 28] in the context of the violent relaxation of $2 \mathrm{D}$ vortices and stellar systems). For $K=+1$, they can provide a dynamical model of the Bose-Einstein condensation in the canonical ensemble which has been studied in detail in [30. When coupled to the field equation (3), the NFP equations (120) and (124) could also provide generalized Keller-Segel models of chemotaxis.

\subsection{Mixed model: anomalous diffusion and filling factor}

The previous models focus individually on two important effects: anomalous diffusion (see Secs. 3.2 3.4) and exclusion constraints when the density becomes too large (see Sec. 3.5). Here we introduce a mixed model which combines these two effects in a single equation. If we take $h(\rho)=q \rho^{q+\mu-1}$ and $g(\rho)=\rho^{\mu+1}\left(1-\rho / \sigma_{0}\right)$, we obtain

$$
\frac{\partial \rho}{\partial t}=\nabla \cdot\left(D q \rho^{q+\mu-1} \nabla \rho+\chi \rho^{\mu+1}\left(1-\rho / \sigma_{0}\right) \nabla \Phi\right) .
$$

This corresponds to a power law diffusion such that $D(\rho)=$ $[D q /(q+\mu)] \rho^{q+\mu-1}$ and a mobility $\chi(\rho)=\chi \rho^{\mu}\left(1-\rho / \sigma_{0}\right)$. The associated stochastic process is

$$
\frac{d \mathbf{r}}{d t}=-\chi \rho^{\mu}\left(1-\rho / \sigma_{0}\right) \nabla \Phi+\sqrt{\frac{2 D q}{q+\mu}} \rho^{\frac{q+\mu-1}{2}} \mathbf{R}(t)
$$

The generalized entropy corresponding to Eq. (126) is obtained by integrating twice the relation

$$
C^{\prime \prime}(\rho)=\frac{q \rho^{q-2}}{1-\rho / \sigma_{0}} .
$$

A first integration gives

$$
C^{\prime}(\rho)=q \sigma_{0}^{q-1} \Phi_{q-2}\left(\frac{\rho}{\sigma_{0}}\right)
$$

where

$$
\Phi_{m}(t)=\int_{0}^{t} \frac{x^{m}}{1-x} d x
$$

Therefore, the generalized entropy can be expressed as

$$
C(\rho)=q \sigma_{0}^{q} \int_{0}^{\rho / \sigma_{0}} \Phi_{q-2}(t) d t .
$$

Note that it does not depend on $\mu$ since the term $\rho^{\mu}$ can be put in factor of the diffusion current in Eq. (126); see the discussion in Sec. 2.10.1
Let us consider some particular cases. (i) For $q=1$, Eq. (126) has the same entropy and the same equilibrium states as Eq. (114). (ii) For $\sigma_{0} \rightarrow+\infty$, we recover Eq. (108). (iii) For $\mu=0$ and $q=2$, we have

$$
\frac{\partial \rho}{\partial t}=\nabla \cdot\left(D \nabla \rho^{2}+\chi \rho\left(1-\rho / \sigma_{0}\right) \nabla \Phi\right) .
$$

The generalized entropy is

$$
S=-2 \sigma_{0}^{2} \int\left(1-\frac{\rho}{\sigma_{0}}\right) \ln \left(1-\frac{\rho}{\sigma_{0}}\right) d \mathbf{r},
$$

and the stationary solution is

$$
\rho=\sigma_{0}\left[1-e^{(\beta \Phi+\alpha) / 2 \sigma_{0}}\right]_{+} .
$$

For $\sigma_{0} \rightarrow+\infty$, we recover Eq. (102). We can also consider the alternative model

$$
\frac{\partial \rho}{\partial t}=\nabla \cdot\left(\frac{2 \rho D}{1-\rho / \sigma_{0}} \nabla \rho+\chi \rho \nabla \Phi\right),
$$

which has the same entropy and the same equilibrium states as Eq. (132). The pressure law is

$$
p(\rho)=-2 T \sigma_{0}^{2}\left[\ln \left(1-\rho / \sigma_{0}\right)-\rho / \sigma_{0}\right] .
$$

(iv) For $(\mu, q)=(0,0)$ and performing the transformation $q D \rightarrow D$, or directly taking $h(\rho)=1 / \rho$ and $g(\rho)=\rho(1-$ $\left.\rho / \sigma_{0}\right)$, we obtain

$$
\frac{\partial \rho}{\partial t}=\nabla \cdot\left(D \nabla \ln \rho+\chi \rho\left(1-\rho / \sigma_{0}\right) \nabla \Phi\right) .
$$

This corresponds to a logarithmic diffusion and a modified mobility taking into account an exclusion principle through the filling factor. The generalized entropy is obtained from the relation

$$
C^{\prime \prime}(\rho)=\frac{1}{\rho^{2}\left(1-\rho / \sigma_{0}\right)},
$$

leading to

$$
C^{\prime}(\rho)=-\frac{1}{\sigma_{0}}\left\{\ln \left(\frac{\sigma_{0}}{\rho}-1\right)+\frac{\sigma_{0}}{\rho}\right\},
$$

and finally to the explicit expression

$$
S=-\int\left(1-\frac{\rho}{\sigma_{0}}\right) \ln \left(\frac{\sigma_{0}}{\rho}-1\right) d \mathbf{r} .
$$

We can consider the alternative model

$$
\frac{\partial \rho}{\partial t}=\nabla \cdot\left[\frac{D}{\rho\left(1-\rho / \sigma_{0}\right)} \nabla \rho+\chi \rho \nabla \Phi\right],
$$

with the same entropy and the same equilibrium states. The associated pressure law is

$$
p(\rho)=-T \ln \left(\frac{\sigma_{0}}{\rho}-1\right) .
$$




\section{$3.8 \kappa$-entropy}

We consider the $\kappa$-entropy

$$
S_{\kappa}=-\frac{1}{2 \kappa} \int\left(\rho^{1+\kappa}-\rho^{1-\kappa}\right) d \mathbf{r} .
$$

This entropy was introduced by Kaniadakis 12 . It can be written

$$
S_{\kappa}=-\int \rho \ln _{(\kappa)} \rho d \mathbf{r}
$$

with the $\kappa$-logarithm

$$
\ln _{(\kappa)}(x)=\frac{1}{2 \kappa}\left(x^{\kappa}-x^{-\kappa}\right) .
$$

We have

$$
\begin{aligned}
& C^{\prime}(\rho)=\frac{1}{2 \kappa}\left[(1+\kappa) \rho^{\kappa}-(1-\kappa) \rho^{-\kappa}\right], \\
& C^{\prime \prime}(\rho)=\frac{1}{2 \rho}\left[(1+\kappa) \rho^{\kappa}+(1-\kappa) \rho^{-\kappa}\right] .
\end{aligned}
$$

If we take $g(\rho)=\rho$ and $h(\rho)=\rho C^{\prime \prime}(\rho)$, we obtain the NFP equation

$$
\frac{\partial \rho}{\partial t}=\nabla \cdot\left(\frac{D}{2} \nabla\left(\rho^{1+\kappa}+\rho^{1-\kappa}\right)+\chi \rho \nabla \Phi\right) .
$$

This corresponds to a power law diffusion $D(\rho)=\frac{D}{2}\left(\rho^{\kappa}+\right.$ $\rho^{-\kappa}$ ) and a constant mobility $\chi(\rho)=\chi$. The associated stochastic process is

$$
\frac{d \mathbf{r}}{d t}=-\chi \nabla \Phi+\sqrt{D}\left(\rho^{\kappa}+\rho^{-\kappa}\right)^{1 / 2} \mathbf{R}(t)
$$

and the stationary solution of Eq. (148) can be written

$$
\rho=\left(\frac{1-\kappa}{1+\kappa}\right)^{\frac{1}{2 \kappa}} e_{(\kappa)}^{-(\beta \Phi+\alpha) / \sqrt{1-\kappa^{2}}}
$$

with the $\kappa$-exponential

$$
e_{(\kappa)}(x)=\left(\kappa x+\sqrt{1+\kappa^{2} x^{2}}\right)^{1 / \kappa} .
$$

Finally, the pressure law is

$$
p(\rho)=\frac{1}{2} T\left(\rho^{1+\kappa}+\rho^{1-\kappa}\right) .
$$

For $\kappa=0$, we recover the standard model 866). These results can be generalized to the $(\kappa, r)$ entropy

$$
S_{\kappa, r}=-\frac{1}{2 \kappa} \int \rho^{r}\left(\rho^{1+\kappa}-\rho^{1-\kappa}\right) d \mathbf{r},
$$

which reduces in some special cases to the Tsallis 9, Abe [10] and Kaniadakis [12] entropies. The corresponding NFP equation can be written

$$
\frac{\partial \rho}{\partial t}=\nabla \cdot\left[\frac{D}{2 \kappa} \nabla\left(a \rho^{1+a}+b \rho^{1-b}\right)+\chi \rho \nabla \Phi\right],
$$

with $a=\kappa+r$ and $b=\kappa-r$. Of course, we could give many other examples of generalized Fokker-Planck equations since there exists an infinite number of distributions and entropic functionals. Therefore, we found it more convenient in 33 to formulate the problem in a general setting, using an arbitrary entropic functional of the form (21).

\section{Nonlinear mean field Fokker-Planck equations in phase space}

We now describe nonlinear mean field Fokker-Planck equations in phase space taking into account the inertia of the particles. Overdamped models will be recovered in a limit of strong friction.

\subsection{Generalized Kramers equation}

We consider a system of $N$ particles in interaction whose dynamics is described by the stochastic Ito-Langevin equations

$$
\begin{gathered}
\frac{d \mathbf{r}_{i}}{d t}=\mathbf{v}_{i} \\
\frac{d \mathbf{v}_{i}}{d t}=-\xi\left(f_{i}\right) \mathbf{v}_{i}-\nabla \Phi_{i}+\sqrt{2 D\left(f_{i}\right)} \mathbf{R}_{i}(t),
\end{gathered}
$$

where $\Phi(\mathbf{r}, t)$ is a self-consistent potential given by the mean field Eq. (2). In ordinary models, the friction $\xi$ and the diffusion coefficient $D$ are constant. In that case, the statistical equilibrium state is the Maxwell-Boltzmann distribution $f \sim e^{-\beta \epsilon}$ where $\epsilon=v^{2} / 2+\Phi(\mathbf{r})$ is the individual energy and the temperature $T=1 / \beta$ is given by the Einstein relation $T=D / \xi$. Here, for sake of generality, the friction coefficient $\xi(f)$ and the diffusion coefficient $D(f)$ are allowed to depend on the distribution function $f(\mathbf{r}, \mathbf{v}, t)=\left\langle\sum_{i=1}^{N} \delta\left(\mathbf{r}-\mathbf{r}_{i}(t)\right) \delta\left(\mathbf{v}-\mathbf{v}_{i}(t)\right)\right\rangle$. This can take into account microscopic constraints that affect the dynamics and modify the equilibrium distribution. The evolution of the distribution function $f(\mathbf{r}, \mathbf{v}, t)$ is governed by the nonlinear mean field Fokker-Planck equation

$$
\frac{\partial f}{\partial t}+\mathbf{v} \cdot \frac{\partial f}{\partial \mathbf{r}}-\nabla \Phi \cdot \frac{\partial f}{\partial \mathbf{v}}=\frac{\partial}{\partial \mathbf{v}} \cdot\left[\frac{\partial}{\partial \mathbf{v}}(D(f) f)+\xi(f) f \mathbf{v}\right]
$$

coupled to Eq. (2). We introduce the notations

$$
D h(f)=\frac{d}{d f}(f D(f)), \quad \xi g(f)=f \xi(f),
$$

where $D$ and $\xi$ are positive constants and $h(f)$ and $g(f)$ are positive functions. The ordinary model with constant diffusion $D(f)=D$ and constant friction $\xi(f)=\xi$ is recovered for $h(f)=1$ and $g(f)=f$. With these notations, 
the NFP equation (157) can be rewritten in the form of a generalized Kramers (GK) equation

$\frac{\partial f}{\partial t}+\mathbf{v} \cdot \frac{\partial f}{\partial \mathbf{r}}-\nabla \Phi \cdot \frac{\partial f}{\partial \mathbf{v}}=\frac{\partial}{\partial \mathbf{v}} \cdot\left(D h(f) \frac{\partial f}{\partial \mathbf{v}}+\xi g(f) \mathbf{v}\right)$.

It can be put in the conservative form

$$
\frac{d f}{d t}=-\frac{\partial}{\partial \mathbf{v}} \cdot \mathbf{J}
$$

where

$$
\mathbf{J}=-\left[D h(f) \frac{\partial f}{\partial \mathbf{v}}+\xi g(f) \mathbf{v}\right]
$$

is a diffusion current. This structure guarantees the conservation of mass $M=\int f d \mathbf{r} d \mathbf{v}$.

\subsection{Generalized free energy and H-theorem}

We define the energy by

$$
E=\frac{1}{2} \int f v^{2} d \mathbf{r} d \mathbf{v}+\frac{1}{2} \int \rho \Phi d \mathbf{r}=K+W
$$

where $K$ is the kinetic energy and $W$ is the potential energy. We define the temperature by

$$
T=\frac{D}{\xi}
$$

The Einstein relation is preserved in the generalized thermodynamical framework. We introduce the generalized entropic functional

$$
S=-\int C(f) d \mathbf{r} d \mathbf{v}
$$

where $C(f)$ is a convex function defined by

$$
C^{\prime \prime}(f)=\frac{h(f)}{g(f)}
$$

Finally, we introduce the generalized free energy

$$
F=E-T S \text {. }
$$

The definition of the free energy (Legendre transform) is preserved in the generalized thermodynamical framework. Explicitly,

$$
F[f]=\frac{1}{2} \int f v^{2} d \mathbf{r} d \mathbf{v}+\frac{1}{2} \int \rho \Phi d \mathbf{r}+T \int C(f) d \mathbf{r} d \mathbf{v} .
$$

A straightforward calculation (see Appendix G) shows that

$$
\dot{F}=-\int \frac{1}{\xi g(f)}\left(D h(f) \frac{\partial f}{\partial \mathbf{v}}+\xi g(f) \mathbf{v}\right)^{2} d \mathbf{r} d \mathbf{v}
$$

Therefore, $\dot{F} \leq 0$. We can also introduce the Massieu function $J=S-\beta E$ which is related to the free energy by $J=-\beta F$. It satisfies $\dot{J} \geq 0$. If $D=0$ (leading to $T=0$ ), we get $F=E$ so that $\dot{E} \leq 0$. If $\chi=0$ (leading to $\beta=0$ ), we get $J=S$ so that $\dot{S} \geq 0$.

\subsection{Stationary solution}

The steady states of Eq. (159) must satisfy $\dot{F}=0$. According to Eq. (168), this implies $\mathbf{J}=\mathbf{0}$ or explicitly

$$
D h(f) \frac{\partial f}{\partial \mathbf{v}}+\xi g(f) \mathbf{v}=\mathbf{0}
$$

Using Eqs. (163) and (165), we get

$$
C^{\prime \prime}(f) \frac{\partial f}{\partial \mathbf{v}}+\beta \mathbf{v}=\mathbf{0}
$$

which can be integrated into

$$
C^{\prime}(f)=-\beta\left[\frac{v^{2}}{2}+\lambda(\mathbf{r})\right],
$$

where $\lambda(\mathbf{r})$ is a function of the position. Since $C$ is convex, this relation can be reversed to give

$$
f(\mathbf{r}, \mathbf{v})=F\left[\beta\left(\frac{v^{2}}{2}+\lambda(\mathbf{r})\right)\right],
$$

where $F(x)=\left(C^{\prime}\right)^{-1}(-x)$ is a decreasing function. Since $\mathbf{J}=\mathbf{0}$ and $\partial f / \partial t=0$, the steady solution of Eq. (159) must also cancel the advective term

$$
\mathbf{v} \cdot \frac{\partial f}{\partial \mathbf{r}}-\nabla \Phi \cdot \frac{\partial f}{\partial \mathbf{v}}=0
$$

In other words, the steady solution of Eq. (159) is a particular stationary solution of the Vlasov equation (l.h.s.) whose form is selected by the "collision" term (r.h.s.). Substituting Eq. (172) in Eq. (173), we get

$$
(\nabla \lambda-\nabla \Phi) \cdot \mathbf{v}=0
$$

which must be true for all $\mathbf{v}$. This yields

$$
\lambda(\mathbf{r})=\Phi(\mathbf{r})+\alpha / \beta,
$$

where $\alpha$ is a constant of integration. Therefore, the stationary solution of Eq. (159) is given by

$$
C^{\prime}(f)=-\beta \epsilon-\alpha
$$

or, equivalently,

$$
f(\mathbf{r}, \mathbf{v})=F[\beta \epsilon(\mathbf{r}, \mathbf{v})+\alpha],
$$

where $\epsilon=v^{2} / 2+\Phi(\mathbf{r})$ is the energy of a particle. Thus, in the steady state, the distribution function $f=f(\epsilon)$ is a function of the individual energy. The potential $\Phi$ is determined by an integrodifferential equation obtained by substituting Eq. (177) in Eq. (2), using $\rho=\int f d \mathbf{v}$. The constant $\alpha$ is determined by the conservation of mass. On the other hand, differentiating Eq. (176), we obtain

$$
\frac{d f}{d \epsilon}=-\frac{\beta}{C^{\prime \prime}(f)} \text {. }
$$

Since $C$ is convex, i.e. $C^{\prime \prime}>0$, the preceding relation implies that $f^{\prime}(\epsilon)<0$. Therefore, $f(\epsilon)$ is a decreasing function of the energy. 


\subsection{Minimum of free energy}

The critical points of free energy at fixed mass are determined by the variational problem

$$
\delta F+T \alpha \delta M=0
$$

where $\alpha$ is a Lagrange multiplier. These variations give

$$
C^{\prime}(f)=-\beta \epsilon-\alpha .
$$

Therefore, comparing with Eq. (176), we find that a stationary solution of Eq. (159) is a critical point of $F$ at fixed mass. Furthermore, it is shown in 33. that a steady state of Eq. (159) is linearly dynamically stable iff it is a minimum (at least local) of $F$ at fixed mass. In this sense, dynamical and generalized thermodynamical stability in the canonical ensemble coincide. This property also results from Lyapunov's direct method 36. Finally, if $F$ is bounded from below, we conclude from the above properties that the system will converge to a stable steady state for $t \rightarrow+\infty$ which is a (local) minimum of $F[f]$ at fixed mass. If several local minima exist, the choice of the final steady state will depend on a complicated notion of basin of attraction. In conclusion, we have the important result: a steady solution of the generalized Kramers equation (159) is linearly dynamically stable iff it is a (local) minimum of the free energy $F[f]$ at fixed mass $M[f]=M$. This corresponds to the minimization problem:

$$
\min _{f}\{F[f] \quad \mid \quad M[f]=M\} .
$$

Taking the second variations of $F$ and using Eq. (178), the condition of dynamical stability is

$$
\delta^{2} F[\delta f]=-\frac{1}{2}\left\{\int \frac{(\delta f)^{2}}{f^{\prime}(\epsilon)} d \mathbf{r} d \mathbf{v}-\int \delta \rho \delta \Phi d \mathbf{r}\right\} \geq 0,
$$

for all perturbations $\delta f$ that conserve mass.

\subsection{Particular cases}

If we take $h(f)=1$ and $g(f)=1 / C^{\prime \prime}(f)$ we get

$$
\frac{\partial f}{\partial t}+\mathbf{v} \cdot \frac{\partial f}{\partial \mathbf{r}}-\nabla \Phi \cdot \frac{\partial f}{\partial \mathbf{v}}=\frac{\partial}{\partial \mathbf{v}} \cdot\left(D \frac{\partial f}{\partial \mathbf{v}}+\frac{\xi}{C^{\prime \prime}(f)} \mathbf{v}\right) .
$$

In that case, we have a constant diffusion $D(f)=D$ and a variable friction $\xi(f)=\xi /\left[f C^{\prime \prime}(f)\right]$. If we take $g(f)=f$ and $h(f)=f C^{\prime \prime}(f)$ we get

$$
\frac{\partial f}{\partial t}+\mathbf{v} \cdot \frac{\partial f}{\partial \mathbf{r}}-\nabla \Phi \cdot \frac{\partial f}{\partial \mathbf{v}}=\frac{\partial}{\partial \mathbf{v}} \cdot\left(D f C^{\prime \prime}(f) \frac{\partial f}{\partial \mathbf{v}}+\xi f \mathbf{v}\right)
$$

In that case, we have a constant friction $\xi(f)=\xi$ and a variable diffusion $D(f)=D f[C(f) / f]^{\prime}$.

\subsection{Functional derivative}

For a given free energy functional $F[f]$, we can introduce phenomenologically a dynamical model by writing the evolution of the distribution function as a continuity equation $d_{t} f=-\frac{\partial}{\partial \mathbf{v}} \cdot \mathbf{J}$ where the current is proportional to the gradient in velocity space of the functional derivative of the free energy, i.e.

$$
\frac{\partial f}{\partial t}+\mathbf{v} \cdot \frac{\partial f}{\partial \mathbf{r}}-\nabla \Phi \cdot \frac{\partial f}{\partial \mathbf{v}}=\frac{\partial}{\partial \mathbf{v}} \cdot\left[\xi(\mathbf{r}, \mathbf{v}, t) f \frac{\partial}{\partial \mathbf{v}} \frac{\delta F}{\delta f}\right] .
$$

For the free energy (167), we have

$$
\frac{\delta F}{\delta f}=T C^{\prime}(f)+\frac{v^{2}}{2}+\Phi
$$

so that

$$
\frac{d f}{d t}=\frac{\partial}{\partial \mathbf{v}} \cdot\left[\xi(\mathbf{r}, \mathbf{v}, t)\left(T f C^{\prime \prime}(f) \frac{\partial f}{\partial \mathbf{v}}+f \mathbf{v}\right)\right]
$$

where we have introduced the material derivative $d / d t=$ $\partial / \partial t+\mathbf{v} \cdot \partial / \partial \mathbf{r}-\nabla \Phi \cdot \partial / \partial \mathbf{v}$ in the advective term. This equation is more general than Eq. (159). It shows that, for a given free energy, we can introduce an infinite class of NFP equations where $\xi(\mathbf{r}, \mathbf{v}, t)$ is an arbitrary positive function of position, velocity and time. In particular, it can be a function of $f(\mathbf{r}, \mathbf{v}, t)$. If we set $\xi(\mathbf{r}, \mathbf{v}, t)=\xi g(f) / f$ we recover Eq. (159). We can also write Eq. (187) in the alternative form

$$
\frac{d f}{d t}=\frac{\partial}{\partial \mathbf{v}} \cdot\left[\tilde{\xi}(\mathbf{r}, \mathbf{v}, t)\left(T \frac{\partial f}{\partial \mathbf{v}}+\frac{1}{C^{\prime \prime}(f)} \mathbf{v}\right)\right]
$$

where $\tilde{\xi}(\mathbf{r}, \mathbf{v}, t)$ is an arbitrary positive function of position, velocity and time. If we set $\tilde{\xi}(\mathbf{r}, \mathbf{v}, t)=\xi h(f)$ we recover Eq. (159). These two alternative forms (187) and (188) were given in 33. On the other hand, the general structure of Eq. (185) implies an $H$-theorem for the free energy $(\dot{F} \leq 0)$. The derivation is similar to that given in Sec. 2.10.1.

\subsection{Equation of state}

The stationary solutions of the nonlinear Kramers equation (159) are of the form $f=f(\epsilon)$ with $f^{\prime}(\epsilon)<0$ where $\epsilon=v^{2} / 2+\Phi(\mathbf{r})$ is the energy of a particle. The function $f$ is determined by the convex function $C$ according to Eq. (176). Therefore, at equilibrium, the density $\rho=\int f d \mathbf{v}$ and the pressure $p=\frac{1}{d} \int f v^{2} d \mathbf{v}$ can be expressed as $\rho=\rho(\Phi(\mathbf{r}))$ and $p=p(\Phi(\mathbf{r}))$. Eliminating the potential $\Phi(\mathbf{r})$ between these expressions, we obtain a barotropic equation of state $p=p(\rho)$ where the function $p(\rho)$ is entirely determined by the convex function $C(f)$. Furthermore, the condition that the distribution function 
is a function $f=f(\epsilon)$ of the energy alone implies the condition of hydrostatic balance. Indeed, we have

$$
\begin{array}{r}
\nabla p=\frac{1}{d} \int f^{\prime}(\epsilon) \nabla \Phi v^{2} d \mathbf{v}=\frac{1}{d} \nabla \Phi \int \frac{\partial f}{\partial \mathbf{v}} \cdot \mathbf{v} d \mathbf{v} \\
=-\nabla \Phi \int f d \mathbf{v}=-\rho \nabla \Phi .
\end{array}
$$

The condition of hydrostatic equilibrium can also be written $p^{\prime}(\Phi)=-\rho(\Phi)$ or

$$
p^{\prime}(\rho)=-\frac{\rho}{\rho^{\prime}(\Phi)} .
$$

Let us introduce the free energy functional

$$
F[\rho]=\int \rho \int^{\rho} \frac{p\left(\rho^{\prime}\right)}{\rho^{\prime 2}} d \rho^{\prime} d \mathbf{r}+\frac{1}{2} \int \rho \Phi d \mathbf{r} .
$$

In Appendix D, we show that this functional of $\rho$ can be deduced from the free energy functional $F[f]$ given by Eq. (166) by using the relation (177) valid at equilibrium. Furthermore, we show in Appendix E that the minimization problem (181) is equivalent to the minimization problem

$$
\min _{\rho}\{F[\rho] \quad \mid \quad M[\rho]=M\}
$$

where $\rho(\mathbf{r})$ is the density profile corresponding to the distribution function $f(\mathbf{r}, \mathbf{v})$. This equivalence considerably simplifies the study of the stability of a steady state of the NFP equation (159). A critical point of $F[\rho]$ at fixed mass satisfies the condition of hydrostatic balance. Indeed, writing $\delta F-\alpha \delta M=0$, we have $\int^{\rho}\left[p^{\prime}\left(\rho^{\prime}\right) / \rho^{\prime}\right] d \rho^{\prime}+\Phi-\alpha=0$ implying $\nabla p+\rho \nabla \Phi=\mathbf{0}$. On the other hand, taking the second variations of $F$ and using Eq. (190), the condition of stability can be written

$$
\delta^{2} F[\delta \rho]=-\frac{1}{2}\left\{\int \frac{(\delta \rho)^{2}}{\rho^{\prime}(\Phi)} d \mathbf{r}-\int \delta \rho \delta \Phi d \mathbf{r}\right\} \geq 0,
$$

for all perturbations $\delta \rho$ that conserve mass.

\subsection{The strong friction limit}

In this section, we shall derive the generalized Smoluchowski equation from the generalized Kramers equation in the strong friction limit $\xi \rightarrow+\infty$. The general case where both the diffusion coefficient and the friction coefficient depend on the distribution function is treated in 54 by using a Chapman-Enskog expansion. Here, we restrict ourselves to the generalized Kramers equation with constant friction coefficient

$$
\frac{\partial f}{\partial t}+\mathbf{v} \cdot \frac{\partial f}{\partial \mathbf{r}}-\nabla \Phi \cdot \frac{\partial f}{\partial \mathbf{v}}=\frac{\partial}{\partial \mathbf{v}} \cdot\left[\xi\left(T f C^{\prime \prime}(f) \frac{\partial f}{\partial \mathbf{v}}+f \mathbf{v}\right)\right] .
$$

In that case, it is possible to develop a procedure simpler that the Chapman-Enskog expansion (see [74]). Let us derive the hierarchy of hydrodynamic equations associated with Eq. (194). Defining the density and the local velocity by

$$
\rho=\int f d \mathbf{v}, \quad \rho \mathbf{u}=\int f \mathbf{v} d \mathbf{v},
$$

and integrating Eq. (194) on velocity, we get the continuity equation

$$
\frac{\partial \rho}{\partial t}+\nabla \cdot(\rho \mathbf{u})=0
$$

Next, multiplying Eq. (194) by $\mathbf{v}$ and integrating on velocity, we obtain the momentum equation

$$
\frac{\partial}{\partial t}\left(\rho u_{i}\right)+\frac{\partial}{\partial x_{j}}\left(\rho u_{i} u_{j}\right)=-\frac{\partial P_{i j}}{\partial x_{j}}-\rho \frac{\partial \Phi}{\partial x_{i}}-\xi \rho u_{i},
$$

where we have defined the pressure tensor

$$
P_{i j}=\int f w_{i} w_{j} d \mathbf{v}
$$

where $\mathbf{w}=\mathbf{v}-\mathbf{u}$ is the relative velocity. Using Eq. (196), the momentum equation can be rewritten in the form

$$
\rho\left(\frac{\partial u_{i}}{\partial t}+u_{j} \frac{\partial u_{i}}{\partial x_{j}}\right)=-\frac{\partial P_{i j}}{\partial x_{j}}-\rho \frac{\partial \Phi}{\partial x_{i}}-\xi \rho u_{i} .
$$

We now consider the strong friction limit $\xi \rightarrow+\infty$ with fixed $T$. Since the term in parenthesis in Eq. (194) must vanish to leading order, we find that the out of equilibrium distribution function $f_{0}(\mathbf{r}, \mathbf{v}, t)$ is given by

$$
C^{\prime}\left(f_{0}\right)=-\beta\left[\frac{v^{2}}{2}+\lambda(\mathbf{r}, t)\right]+O\left(\xi^{-1}\right),
$$

where $\lambda(\mathbf{r}, t)$ is a constant of integration that is determined by the density according to

$$
\rho(\mathbf{r}, t)=\int f_{0} d \mathbf{v}=\rho[\lambda(\mathbf{r}, t)] .
$$

Note that the distribution function $f_{0}$ is isotropic so that the velocity $\mathbf{u}(\mathbf{r}, t)=O\left(\xi^{-1}\right)$ and the pressure tensor $P_{i j}=p \delta_{i j}+O\left(\xi^{-1}\right)$ where $p$ is given by

$$
p(\mathbf{r}, t)=\frac{1}{d} \int f_{0} v^{2} d \mathbf{v}=p[\lambda(\mathbf{r}, t)] .
$$

Eliminating $\lambda(\mathbf{r}, t)$ between the two expressions (201) and (202), we find that the fluid is barotropic with an equation of state $p=p(\rho)$ entirely determined by the function $C(f)$. Of course, this is the same equation of state as the one obtained at equilibrium (see Sec. 4.7). Now, considering the momentum equation (197) in the limit $\xi \rightarrow+\infty$, we find that

$$
\rho \mathbf{u}=-\frac{1}{\xi}(\nabla p+\rho \nabla \Phi)+O\left(\xi^{-2}\right) .
$$


Inserting this relation in the continuity equation (196), we obtain the generalized Smoluchowski equation 337454:

$$
\frac{\partial \rho}{\partial t}=\nabla \cdot\left[\frac{1}{\xi}(\nabla p+\rho \nabla \Phi)\right]
$$

The free energy associated to this equation is

$$
F[\rho]=\int \rho \int^{\rho} \frac{p\left(\rho^{\prime}\right)}{\rho^{\prime 2}} d \rho^{\prime} d \mathbf{r}+\frac{1}{2} \int \rho \Phi d \mathbf{r}
$$

It can be deduced from the free energy (166) by using Eq. (200) to express $F[f]$ as a functional $F[\rho]=F\left[f_{0}\right]$ of the density (see Appendix (D). A direct calculation leads to the $H$-theorem

$$
\dot{F}=-\int \frac{1}{\xi \rho}(\nabla p+\rho \nabla \Phi)^{2} d \mathbf{r} \leq 0
$$

The stationary solutions of the generalized Smoluchowski equation (204) are critical points of free energy at fixed mass. They satisfy the condition of hydrostatic balance

$$
\nabla p+\rho \nabla \Phi=\mathbf{0}
$$

From Lyapunov's direct method, we conclude that a steady state of the generalized Smoluchowski equation (204) is linearly dynamically stable iff it is a (local) minimum of $F$ at fixed mass $M$. This corresponds to the minimization problem (192).

The condition of hydrostatic balance (207) only holds at equilibrium. In the strong friction limit $\xi \rightarrow+\infty$, the out-of-equilibrium distribution is of the form $f_{0}=f_{0}\left(\epsilon^{\prime}\right)$ with $\epsilon^{\prime}=v^{2} / 2+\lambda(\mathbf{r}, t)$. Taking the gradient of Eq. (202) and using a procedure similar to that followed in Eq. (189) with $\lambda(\mathbf{r}, t)$ in place of $\Phi(\mathbf{r})$, we obtain

$$
\nabla p=-\rho \nabla \lambda
$$

Since $p=p(\rho)$ and $\lambda=\lambda(\rho)$, this can be rewritten

$$
\lambda^{\prime}(\rho)=-\frac{p^{\prime}(\rho)}{\rho}
$$

so that the out-of-equilibrium chemical potential $\lambda(\mathbf{r}, t)$ is given by

$$
\lambda(\rho)=-\int^{\rho} \frac{p^{\prime}(x)}{x} d x
$$

At equilibrium, comparing Eqs. (208) and (207), we have $\lambda(\mathbf{r})=\Phi(\mathbf{r})+\alpha / \beta$ and Eq. (200) leads to Eq. (176).

\subsection{The damped Euler equations}

The generalized Smoluchowski equation (204) can also be obtained formally from the damped Euler 7 equations 33 :

$$
\frac{\partial \rho}{\partial t}+\nabla \cdot(\rho \mathbf{u})=0
$$

$$
\frac{\partial \mathbf{u}}{\partial t}+(\mathbf{u} \cdot \nabla) \mathbf{u}=-\frac{1}{\rho} \nabla p-\nabla \Phi-\xi \mathbf{u}
$$

The Lyapunov functional associated with the damped Euler equations is the generalized free energy

$$
F[\rho, \mathbf{u}]=\int \rho \int^{\rho} \frac{p\left(\rho^{\prime}\right)}{\rho^{\prime 2}} d \rho^{\prime} d \mathbf{r}+\frac{1}{2} \int \rho \Phi d \mathbf{r}+\int \rho \frac{\mathbf{u}^{2}}{2} d \mathbf{r} .
$$

It satisfies an $H$-theorem of the form (see Appendix $\mathrm{G}$ ):

$$
\dot{F}=-\int \xi \rho \mathbf{u}^{2} d \mathbf{r} \leq 0 .
$$

A steady state of the damped Euler equations (211)-(212) satisfies the condition of hydrostatic equilibrium (207). Furthermore, from Lyapunov's direct method, it is linearly dynamically stable iff it is a minimum of the free energy (213) at fixed mass. This corresponds to the minimization problem

$$
\min _{\rho, \mathbf{u}}\{F[\rho, \mathbf{u}] \quad \mid \quad M[\rho]=M\}
$$

The friction coefficient in Eq. (212) measures the importance of inertial effects. For $\xi=0$, we recover the usual barotropic Euler equations. Alternatively, if we consider the strong friction limit $\xi \rightarrow+\infty$, we can neglect the inertial term in Eq. (212) and we get

$$
\xi \mathbf{u}=-\frac{1}{\rho} \nabla p-\nabla \Phi+O\left(\xi^{-1}\right) .
$$

Substituting this relation in the continuity equation (211), we obtain the generalized Smoluchowski equation

$$
\frac{\partial \rho}{\partial t}=\nabla \cdot\left[\frac{1}{\xi}(\nabla p+\rho \nabla \Phi)\right] .
$$

The physical justification of the damped Euler equations (211)-212 is not clear. They can be obtained from Eqs. (196)-1197) if we close the hierarchy by invoking a local thermodynamic equilibrium (L.T.E.) condition [33. However, the rigorous justification of this L.T.E. condition is

\footnotetext{
7 Hydrodynamical Euler equations involving a friction force $-\xi(|\mathbf{u}|) \mathbf{u}$ have appeared in various contexts for different reasons. We may mention, for example, bottom-wall friction in 2D turbulence [75], frictional force of air on a turbulent soap film 76, effective dynamical friction in the process of violent relaxation for collisionless stellar systems 28] and Epstein or Stokes friction laws for the dynamics of dust particles in the solar nebula 77.
} 
not established, so this approach remains heuristic. Nevertheless, hydrodynamic equations (hyperbolic models) of the form (211)-(212) have been proposed in the context of chemotaxis to describe the organization of endothelial cells 78179151. They lead to the formation of filaments that are interpreted as the beginning of a vasculature. These filaments are not obtained in the Keller-Segel model (parabolic model) which leads to point-wise blow up or round aggregates $52[42$.

\section{Explicit examples}

In this section, we give explicit examples showing the passage from the generalized Kramers equation to the generalized Smoluchowski equation in the strong friction limit.

\subsection{Isothermal systems: Boltzmann entropy}

If we consider the Boltzmann entropy

$$
S_{B}[f]=-\int f \ln f d \mathbf{r} d \mathbf{v},
$$

we get the ordinary Kramers equation

$$
\frac{d f}{d t}=\frac{\partial}{\partial \mathbf{v}} \cdot\left[\xi\left(T \frac{\partial f}{\partial \mathbf{v}}+f \mathbf{v}\right)\right] .
$$

The stationary state is the isothermal (Maxwell-Boltzmann) distribution

$$
f=A e^{-\beta \epsilon},
$$

where $A$ is determined by the conservation of mass. The equation of state is the isothermal one

$$
p=\rho T \text {. }
$$

In the strong friction limit, we obtain the ordinary Smoluchowski equation

$$
\frac{\partial \rho}{\partial t}=\nabla \cdot\left[\frac{1}{\xi}(T \nabla \rho+\rho \nabla \Phi)\right] .
$$

The corresponding free energy is the Boltzmann free energy

$$
F[\rho]=T \int \rho \ln \rho d \mathbf{r}+\frac{1}{2} \int \rho \Phi d \mathbf{r},
$$

and the stationary solution is the Boltzmann distribution

$$
\rho=A^{\prime} e^{-\beta \Phi}
$$

where $A^{\prime}=(2 \pi / \beta)^{d / 2} A$.

\subsection{Polytropes: Tsallis entropy}

If we consider the Tsallis $q$-entropy

$$
S_{q}[f]=-\frac{1}{q-1} \int\left(f^{q}-f\right) d \mathbf{r} d \mathbf{v},
$$

we get the polytropic Kramers equation

$$
\frac{d f}{d t}=\frac{\partial}{\partial \mathbf{v}} \cdot\left[\xi\left(T \frac{\partial f^{q}}{\partial \mathbf{v}}+f \mathbf{v}\right)\right] .
$$

The stationary state is the polytropic distribution

$$
f=\left[\mu-\frac{(q-1) \beta}{q} \epsilon\right]_{+}^{\frac{1}{q-1}},
$$

where $\mu$ is determined by the conservation of mass. The index $n$ of the polytrope is related to the parameter $q$ by the relation

$$
n=\frac{d}{2}+\frac{1}{q-1} .
$$

Isothermal distribution functions are recovered in the limit $q \rightarrow 1$ (i.e. $n \rightarrow+\infty$ ). We shall consider $q>0$ so that $C$ is convex. We have to distinguish two cases. (i) For $q>1$, i.e. $n>d / 2$, the distribution has a compact support since $f$ is defined only for $\epsilon \leq \epsilon_{m} \equiv \mu q /[|q-1| \beta]$ (it vanishes at $\left.\epsilon=\epsilon_{m}\right)$. For $\epsilon \geq \epsilon_{m}$, we set $f=0$. For $q \rightarrow+\infty$, i.e. $n=d / 2, f$ is the Heaviside function. (ii) For $q<1$, the distribution is defined for all energies. For large velocities, it behaves like $f \sim v^{-(d-2 n)}$. Therefore, the density and the pressure are finite only for $n<-1$, i.e. $d /(d+2)<$ $q<1$. Therefore the range of allowed parameters are

$$
\begin{gathered}
q>1, \quad n>\frac{d}{2} \quad(\text { case } 1), \\
\frac{d}{d+2}<q<1, \quad n<-1 \quad(\text { case } 2) .
\end{gathered}
$$

The distribution function (227) leads to the polytropic equation of state (see Appendix $\underline{\mathrm{H}}$ )

$$
p=K \rho^{\gamma}, \quad \gamma=1+\frac{1}{n} .
$$

For $n>d / 2$ the polytropic constant is

$$
K=\frac{1}{n+1}\left[A S_{d} 2^{\frac{d}{2}-1} \frac{\Gamma(d / 2) \Gamma(1-d / 2+n)}{\Gamma(1+n)}\right]^{-1 / n},
$$

and for $n<-1$, we have

$$
K=-\frac{1}{n+1}\left[A S_{d} 2^{\frac{d}{2}-1} \frac{\Gamma(d / 2) \Gamma(-n)}{\Gamma(d / 2-n)}\right]^{-1 / n},
$$

where $A=(\beta|q-1| / q)^{1 /(q-1)}$. In the strong friction limit, we get the polytropic Smoluchowski equation

$$
\frac{\partial \rho}{\partial t}=\nabla \cdot\left[\frac{1}{\xi}\left(K \nabla \rho^{\gamma}+\rho \nabla \Phi\right)\right] .
$$


The generalized free energy is the Tsallis free energy

$$
F[\rho]=\frac{K}{\gamma-1} \int\left(\rho^{\gamma}-\rho\right) d \mathbf{r}+\frac{1}{2} \int \rho \Phi d \mathbf{r},
$$

and the stationary solution is the polytropic distribution

$$
\rho=\left[\lambda-\frac{\gamma-1}{K \gamma} \Phi\right]^{\frac{1}{\gamma-1}}
$$

Other useful relations valid for polytropic distributions are given in 80. We note that a polytropic distribution with index $q$ in phase space yields a polytropic distribution with index $\gamma=1+2(q-1) /[2+d(q-1)]$ in physical space. In this sense, Tsallis distributions are stable laws since the functions $f(\epsilon)$ and $\rho(\Phi)$ have a similar structure. By comparing Eq. (227) to Eq. (236) or Eq. (166) with Eq. (225) to Eq. 235), we note that $K$ plays the same role in physical space as the temperature $T=1 / \beta$ in phase space. It is sometimes called a "polytropic temperature".

Quite generally, we define the local kinetic temperature $T(\mathbf{r})$ by $\frac{d}{2} T(\mathbf{r})=\frac{1}{2}\left\langle v^{2}\right\rangle$ or equivalently $p(\mathbf{r})=\rho(\mathbf{r}) T(\mathbf{r})$. It is proportional to the velocity dispersion. Since, at equilibrium, $\rho=\rho(\Phi)$ and $p=p(\Phi)$, we conclude that $T(\mathbf{r})=$ $T[\Phi(\mathbf{r})]$ is a function of the potential $\Phi$. This is true for any barotropic fluid at equilibrium. Now, for a polytropic distribution, using Eq. (231), we have $T(\mathbf{r})=K \rho^{\gamma-1}$. Then, using Eq. 236 we obtain $T(\mathbf{r})=K \lambda-\frac{\gamma-1}{\gamma} \Phi(\mathbf{r})$ so that $T$ is a linear function of $\Phi$ with a gradient $(\gamma-1) / \gamma=$ $1 /(n+1)=2(q-1) /[(d+2) q-d]$ [80 81].

\subsection{Fermions: Fermi-Dirac entropy}

If we consider the Fermi-Dirac entropy

$S_{F D}[f]=-\eta_{0} \int\left\{\frac{f}{\eta_{0}} \ln \frac{f}{\eta_{0}}+\left(1-\frac{f}{\eta_{0}}\right) \ln \left(1-\frac{f}{\eta_{0}}\right)\right\} d \mathbf{r} d \mathbf{v}$

we get the fermionic Kramers equation

$$
\frac{d f}{d t}=\frac{\partial}{\partial \mathbf{v}} \cdot\left[\xi\left(-T \eta_{0} \frac{\partial}{\partial \mathbf{v}} \ln \left(1-\frac{f}{\eta_{0}}\right)+f \mathbf{v}\right)\right] .
$$

The corresponding equation with normal diffusion and nonlinear friction is

$$
\frac{d f}{d t}=\frac{\partial}{\partial \mathbf{v}} \cdot\left[D\left(\frac{\partial f}{\partial \mathbf{v}}+\beta f\left(1-f / \eta_{0}\right) \mathbf{v}\right)\right]
$$

The stationary state is the Fermi-Dirac distribution function

$$
f=\frac{\eta_{0}}{1+\lambda e^{\beta \epsilon}}
$$

where $\lambda>0$ is determined by the conservation of mass. The Fermi-Dirac distribution function (240) satisfies the constraint $f \leq \eta_{0}$ which is related to the Pauli exclusion principle in quantum mechanics. The isothermal distribution function (220) is recovered in the non-degenerate limit $f \ll \eta_{0}$. This is the case when $\lambda \rightarrow+\infty$, valid at high temperatures $T \rightarrow+\infty$. On the other hand, in the completely degenerate limit, the distribution is a step function $f=\eta_{0} H\left(\epsilon-\epsilon_{F}\right)$ where $\epsilon_{F}=-\frac{1}{\beta} \ln \lambda$ is the Fermi energy. This is the case when $\lambda \rightarrow 0$, valid at low temperatures $T \rightarrow 0$. This limiting distribution corresponds to a polytrope with index $n=d / 2$. The distribution in physical space, obtained by integrating the Fermi-Dirac statistics (240) on the velocity, can be written

$$
\rho=\frac{\eta_{0} S_{d} 2^{\frac{d}{2}-1}}{\beta^{d / 2}} I_{\frac{d}{2}-1}\left(\lambda e^{\beta \Phi}\right)
$$

where $I_{n}$ is the Fermi integral

$$
I_{n}(t)=\int_{0}^{+\infty} \frac{x^{n}}{1+t e^{x}} d x .
$$

The quantum equation of state for fermions is given in parametric form by

$$
\rho=\frac{\eta_{0} S_{d} 2^{\frac{d}{2}-1}}{\beta^{d / 2}} I_{\frac{d}{2}-1}(t), \quad p=\frac{\eta_{0} S_{d} 2^{\frac{d}{2}}}{d \beta^{\frac{d}{2}+1}} I_{\frac{d}{2}}(t) .
$$

At high temperatures $(t \rightarrow+\infty)$ we recover the classical isothermal law $p=\rho T$ and at low temperatures $(t \rightarrow 0)$ we get a polytropic equation of state $p=K \rho^{\gamma}$ with $\gamma=(d+2) / 2$ (i.e. $n=d / 2)$ and $K=\frac{1}{d+2}\left(\frac{d}{\eta_{0} S_{d}}\right)^{2 / d}$ [82. In the strong friction limit, we get the fermionic Smoluchowski equation (204) where the equation of state is given by (243). The fermionic Smoluchowski-Poisson system has been studied in 286683.

\section{Conclusion}

In this paper, we have studied a general class of nonlinear mean field Fokker-Planck equations 33 associated with a formalism of effective generalized thermodynamics (E.G.T.). We have given several physical examples of application and we have shown that NFP equations can provide generalizations of the standard Keller-Segel model describing the chemotaxis of biological populations. The main properties of these NFP equations are valid for a large class of entropic functionals, encompassing the Boltzmann, the Fermi-Dirac, the Bose-Einstein and the Tsallis statistics. Indeed, the rich mathematical structure of these equations is almost independent on the precise form of the entropy. These results should therefore evidence which properties in statistical mechanics are specific to the standard Boltzmann entropy and which properties are valid for a larger class of entropies. The distinguished feature of the Boltzmann entropy is that it can be obtained from a combinatorial analysis, assuming that all the microstates are equiprobable. However, equiprobability of the microstates is a strong postulate and it is not clear whether it has a universal scope. The universality of the Boltzmann entropy has been criticized long ago by Einstein 84 who argued that the statistics applicable on a system depends on its underlying dynamics (see discussion in [85]). For example, the Boltzmann distribution can 
be obtained from a stochastic process describing a classical random walk where the kicks have uniform sizes. However, different distributions emerge when the stochastic process describes a biased random walk where the kicks depend on the region where the particle happens to be. We think that this is the case in many physical systems. This results in a very complex geometrical structure of phase space (fractal, multi-fractal,...) leading to non-Boltzmannian distributions at equilibrium. Indeed, in such circumstances, the microstates are not equiprobable since the system prefers certain regions of phase space rather than others. It would be interesting to derive the corresponding generalized entropies (Tsallis, Abe, Kaniadakis,...) directly from a combinatorial analysis. For example, Tsallis entropy could be the natural entropy on a fractal phase-space. The selection of the entropy demands a complete specification of the microdynamics of the system in agreement with the statement given long ago by Einstein [84].

\section{A The isotropic BGK operator}

Let us consider a simple kinetic equation where the generalized Fokker-Planck operator in Eq. (159) is replaced by a generalized isotropic BGK operator. This equation has been introduced in Appendix A of [54 and we provide here some complements. The kinetic equation is

$$
\frac{\partial f}{\partial t}+\mathbf{v} \cdot \frac{\partial f}{\partial \mathbf{r}}-\nabla \Phi \cdot \frac{\partial f}{\partial \mathbf{v}}=-\frac{f-f_{0}}{\tau}
$$

where $f_{0}(\mathbf{r}, \mathbf{v}, t)$ is given by

$$
C^{\prime}\left(f_{0}\right)=-\beta\left[\frac{v^{2}}{2}+\lambda(\mathbf{r}, t)\right]
$$

or

$$
f_{0}=F\left\{\beta\left[\frac{v^{2}}{2}+\lambda(\mathbf{r}, t)\right]\right\}
$$

with $F(x)=\left(C^{\prime}\right)^{-1}(-x)$. The function $\lambda(\mathbf{r}, t)$ is determined by the density by writing $\rho=\int f_{0} d \mathbf{v}=\rho(\lambda)$. First, we show that Eq. (244) admits an H-theorem for the free energy (166). Recalling that the left hand side (Vlasov term) conserves the energy and the Casimirs, hence $F$, we find that

$$
\dot{F}=-\int\left(T C^{\prime}(f)+\frac{v^{2}}{2}\right) \frac{f-f_{0}}{\tau} d \mathbf{r} d \mathbf{v} .
$$

Using Eq. (245) and the fact that $\int f_{0} d \mathbf{v}=\int f d \mathbf{v}$, we have the identity

$$
\begin{aligned}
& \int\left(T C^{\prime}\left(f_{0}\right)+\frac{v^{2}}{2}\right) \frac{f-f_{0}}{\tau} d \mathbf{r} d \mathbf{v} \\
& =-\beta \int \lambda(\mathbf{r}, t) \frac{f-f_{0}}{\tau} d \mathbf{r} d \mathbf{v}=0 .
\end{aligned}
$$

Therefore, we can rewrite Eq. (247) in the form

$$
\dot{F}=-T \int\left[C^{\prime}(f)-C^{\prime}\left(f_{0}\right)\right] \frac{f-f_{0}}{\tau} d \mathbf{r} d \mathbf{v} .
$$

Since $C$ is convex, we have

$$
\left[C^{\prime}(f)-C^{\prime}\left(f_{0}\right)\right]\left(f-f_{0}\right) \geq 0,
$$

so that $\dot{F} \leq 0$. On the other hand, using a procedure similar to that described in Sec. 4.3, it is straightforward to prove that the steady states of Eq. (244) are given by Eq. (176) and that a steady state of Eq. (244) is dynamically stable iff it is a (local) minimum of $F$ at fixed mass. The first two hydrodynamic equations associated with Eq. (244) are

$$
\begin{gathered}
\frac{\partial \rho}{\partial t}+\nabla \cdot(\rho \mathbf{u})=0 \\
\frac{\partial}{\partial t}\left(\rho u_{i}\right)+\frac{\partial}{\partial x_{j}}\left(\rho u_{i} u_{j}\right)=-\frac{\partial P_{i j}}{\partial x_{j}}-\rho \frac{\partial \Phi}{\partial x_{i}}-\frac{1}{\tau} \rho u_{i} .
\end{gathered}
$$

In the limit $\tau \rightarrow 0$, we can repeat the same arguments as in Sec. 4.8 and we obtain the generalized Smoluchowski equation

$$
\frac{\partial \rho}{\partial t}=\nabla \cdot[\tau(\nabla p+\rho \nabla \Phi)] .
$$

This equation can also be obtained from a ChapmanEnskog expansion [54. Rigorous mathematical results have been obtained recently in [86.

\section{B Connection between dynamical and thermodynamical stability for nonlinear mean field Fokker-Planck equations}

Let us consider a small perturbation $\delta \rho$ around a stationary solution of the nonlinear mean field Fokker-Planck equation (87). We write the time dependence of the perturbation as $\delta \rho \sim e^{\lambda t}$. It can be shown that $\lambda$ is real, so that the perturbation is damped exponentially for $\lambda<0$ (stable) or increases exponentially for $\lambda>0$ (unstable). The linearized Fokker-Planck equation can be written

$$
\lambda \delta \rho=-\nabla \cdot \delta \mathbf{J} .
$$

If we multiply Eq. (254) by $C^{\prime \prime}(\rho) \delta \rho$ and integrate the resulting expression over the volume, we get

$\lambda \int C^{\prime \prime}(\rho)(\delta \rho)^{2} d \mathbf{r}=\int \delta \mathbf{J} \cdot\left[C^{\prime \prime}(\rho) \nabla \delta \rho+C^{\prime \prime \prime}(\rho) \delta \rho \nabla \rho\right] d \mathbf{r}$,

where we have used an integration by parts in the r.h.s. Now, the linear variation of the current (10) around equilibrium can be written

$-\frac{\delta \mathbf{J}}{D}=h^{\prime}(\rho) \delta \rho \nabla \rho+h(\rho) \nabla \delta \rho+\beta g^{\prime}(\rho) \delta \rho \nabla \Phi+\beta g(\rho) \nabla \delta \Phi$. 
Using $h(\rho)=C^{\prime \prime}(\rho) g(\rho)$ and the relation

$$
C^{\prime \prime}(\rho) \nabla \rho=-\beta \nabla \Phi,
$$

resulting from Eq. (29), we obtain

$$
-\frac{\delta \mathbf{J}}{D g(\rho)}=C^{\prime \prime \prime}(\rho) \delta \rho \nabla \rho+C^{\prime \prime}(\rho) \nabla \delta \rho+\beta \nabla \delta \Phi .
$$

Then, Eq. 255 can be rewritten

$$
\lambda \int C^{\prime \prime}(\rho)(\delta \rho)^{2} d \mathbf{r}+\beta \int \delta \mathbf{J} \cdot \nabla \delta \Phi d \mathbf{r}=-\int \frac{(\delta \mathbf{J})^{2}}{D g(\rho)} d \mathbf{r}
$$

Now, multiplying Eq. (254) by $\delta \Phi$ and integrating over the volume we find that

$$
\lambda \int \delta \rho \delta \Phi d \mathbf{r}=\int \delta \mathbf{J} \cdot \nabla \delta \Phi d \mathbf{r},
$$

where we have used an integration by parts in the r.h.s. Inserting Eq. (260) in Eq. (259), we obtain

$$
-\lambda \beta \int \frac{(\delta \rho)^{2}}{\rho^{\prime}(\Phi)} d \mathbf{r}+\lambda \beta \int \delta \rho \delta \Phi d \mathbf{r}=-\int \frac{(\delta \mathbf{J})^{2}}{D g(\rho)} d \mathbf{r} .
$$

On the other hand, the second variations of the rate of free energy dissipation (24) around equilibrium are

$$
\delta^{2} \dot{F}=-\int \frac{(\delta \mathbf{J})^{2}}{\beta D g(\rho)} d \mathbf{r},
$$

and they are clearly negative. Inserting Eqs. (262) and (40) in Eq. (261), we finally obtain the relation

$$
2 \lambda \delta^{2} F=\delta^{2} \dot{F} \leq 0 .
$$

This relation shows that a steady state of the nonlinear mean field Fokker-Planck equation (8) is linearly dynamically stable $(\lambda<0)$ iff it is a minimum (at least local) of the free energy at fixed mass $\left(\delta^{2} F>0\right)$. Therefore, dynamical and generalized thermodynamical stability coincide.

\section{Stability of the homogeneous phase and critical point}

We consider a homogeneous stationary solution $\rho(\mathbf{r})=\rho$ of the nonlinear mean field Fokker-Planck equation (8). The corresponding potential is $\Phi(\mathbf{r})=\Phi=U \rho$ where $U \equiv \int u(\mathbf{x}) d \mathbf{x}$. The dynamical evolution of a small perturbation around equilibrium is given by the linearized equation

$$
\begin{aligned}
\frac{\partial \delta \rho}{\partial t} & =\nabla \cdot[D h(\rho) \nabla \delta \rho+\chi g(\rho) \nabla \delta \Phi] \\
& =D g(\rho)\left[C^{\prime \prime}(\rho) \Delta \delta \rho+\beta \Delta \delta \Phi\right],
\end{aligned}
$$

where we have used Eqs. (20) and (22) to get the second line. The perturbations $\delta \rho$ and $\delta \Phi$ can be decomposed in Fourier modes of the form $f(\mathbf{r}, t)=\int \hat{f}(\mathbf{k}) e^{i \mathbf{k} \cdot \mathbf{r}} e^{\lambda(\mathbf{k}) t} d \mathbf{k}$ leading to the dispertion relation

$$
\lambda(\mathbf{k})=-D g(\rho) k^{2}\left[C^{\prime \prime}(\rho)-\beta \hat{v}(k)\right],
$$

where we have used $\delta \hat{\Phi}=(2 \pi)^{d} \hat{u}(k) \delta \hat{\rho}$ (convolution) and set $\hat{v}(k)=-(2 \pi)^{d} \hat{u}(k)$. The system is stable $(\lambda<0)$ if

$$
C^{\prime \prime}(\rho)-\beta \hat{v}(k)>0,
$$

for all $k$ and unstable (to some wavenumbers) otherwise. For a potential satisfying $\hat{v}<0$, the homogeneous phase is always stable. Otherwise, there exists a critical point in the problem. The homogeneous phase is stable for

$$
T>T_{c} \equiv \frac{\hat{v}(k)_{\max }}{C^{\prime \prime}(\rho)},
$$

where $\hat{v}(k)_{\max }$ is the largest value of $\hat{v}(k)$ achieved for $k=$ $k_{*}$. On the other hand, for $T<T_{c}$ the homogeneous phase is unstable to wavenumbers satisfying $\hat{v}(k)>T C^{\prime \prime}(\rho)$. The growth rate of the mode $k$ is given by Eq. (265). This stability analysis has been explicited for particular potentials of the form (2) in [40 87. It has also been generalized to potentials of the form (3) in 88.

The condition of generalized thermodynamical stability demands that $\rho$ is a minimum of the free energy $F[\rho]$, given by Eq. (36), at fixed mass. Using Eq. (31), the stability criterion (40) can be rewritten

$$
\delta^{2} F=\frac{1}{2 \beta}\left\{\int C^{\prime \prime}(\rho)(\delta \rho)^{2} d \mathbf{r}+\beta \int \delta \rho \delta \Phi d \mathbf{r}\right\} \geq 0,
$$

for all perturbations $\delta \rho$ that conserve mass. We need therefore to investigate the eigenvalue equation

$$
C^{\prime \prime}(\rho) \delta \rho+\beta \delta \Phi=\lambda \delta \rho .
$$

The homogeneous phase is a minimum of free energy at fixed mass (stable) iff all the eigenvalues $\lambda$ are positive. If at least one eigenvalue is negative, the homogeneous phase is an unstable saddle point of free energy at fixed mass. Solving the eigenvalue Eq. (269) in Fourier space, we get

$$
C^{\prime \prime}(\rho)-\beta \hat{v}(k)=\lambda .
$$

The spectrum of eiganvalues is continuous and Eq. (270) determines the eigenmode $k$ corresponding to the eigenvalue $\lambda$. Therefore, the homogeneous phase is a minimum of free energy at fixed mass (stable) iff the 1.h.s. of Eq. (270) is positive for all $k$. This returns the condition (266), i.e. $T>T_{c}$. Alternatively, for $T<T_{c}$, there exists modes $k$ such that $\lambda<0$ implying $\delta^{2} F<0$ for these modes. In that case, the homogeneous phase is a maximum or a saddle point of free energy at fixed mass (unstable). Using the Parseval theorem, the second variations of free energy (268) can be written

$$
\delta^{2} F=\frac{1}{2 \beta}(2 \pi)^{d} \int\left[C^{\prime \prime}(\rho)-\beta \hat{v}(k)\right]|\delta \hat{\rho}|^{2} d \mathbf{k},
$$


leading directly to the preceding results. We also check on this explicit example (homogeneous state) that the conditions of dynamical and generalized thermodynamical stability coincide. As shown in Appendix B, this is also true for inhomogeneous equilibrium distributions.

Finally, let us show that $T_{c}$ corresponds to a bifurcation point. The general steady state of the nonlinear mean field Fokker-Planck equation (8) is given by Eq. (29). Close to the bifurcation point, the inhomogeneous equilibrium density profile can be written $\rho(\mathbf{r})=\rho+\epsilon(\mathbf{r})$ where $\rho$ is the homogeneous solution and $\epsilon(\mathbf{r}) \ll \rho$. Substituting this relation in Eq. (29) and expanding the equation to first order in $\epsilon$, we get

$$
C^{\prime \prime}(\rho) \epsilon(\mathbf{r})=-\beta \int \epsilon\left(\mathbf{r}^{\prime}\right) u\left(\left|\mathbf{r}-\mathbf{r}^{\prime}\right|\right) d \mathbf{r}^{\prime} .
$$

In Fourier space, this relation becomes

$$
C^{\prime \prime}(\rho) \hat{\epsilon}(\mathbf{k})=\beta \hat{v}(k) \hat{\epsilon}(\mathbf{k}) .
$$

This equation has a non-zero solution $\hat{\epsilon}(\mathbf{k}) \neq 0$ provided that there exists a mode $k=k_{*}$ such that $C^{\prime \prime}(\rho)=\beta \hat{v}\left(k_{*}\right)$. This precisely corresponds to the condition $T=T_{c}$. Therefore, stable stationary inhomogeneous solutions appear for $T<T_{c}$ precisely when the homogeneous phase becomes unstable. For the BMF model [4], the stability analysis can be carried out explicitly and we find that the phase transition at $T=T_{c}$ is second order. More generally, there may exist other systems where inhomogeneous solutions are stable for $T>T_{c}$. In that case, they are in "competition" with the homogeneous solution. One of these solutions is stable (global minimum of free energy) and the other metastable (local minimum of free energy). This is usually associated with a first order phase transition.

\section{Passage from $F[f]$ to $F[\rho]$}

We assume that the distribution function $f(\mathbf{r}, \mathbf{v}, t)$ is given by an expression of the form

$$
C^{\prime}(f)=-\beta\left[\frac{v^{2}}{2}+\lambda(\mathbf{r}, t)\right] .
$$

This expression appeared at several occasions in our analysis (see Secs. 4.3, $4.7,4.8$ and Appendix E). Since $C$ is convex, the foregoing relation can be reversed to give

$$
f=F\left[\beta\left(\frac{v^{2}}{2}+\lambda(\mathbf{r}, t)\right)\right]
$$

where $F(x)=\left(C^{\prime}\right)^{-1}(-x)$. Since the distribution function is isotropic, the local velocity vanishes: $\mathbf{u}=\mathbf{0}$. The density and the pressure are then given by

$$
\rho=\int f d \mathbf{v}=\rho[\lambda], \quad p=\frac{1}{d} \int f v^{2} d \mathbf{v}=p[\lambda] .
$$

The first relation determines $\lambda(\mathbf{r}, t)$ as a function of the density $\rho(\mathbf{r}, t)$. Substituting Eq. (275) in Eq. (276), we find that

$$
\rho=\frac{1}{\beta^{d / 2}} g(\beta \lambda), \quad p=\frac{1}{\beta^{\frac{d+2}{2}}} h(\beta \lambda),
$$

with

$$
\begin{array}{r}
g(x)=2^{\frac{d-2}{2}} S_{d} \int_{0}^{+\infty} F(x+t) t^{\frac{d-2}{2}} d t, \\
h(x)=\frac{1}{d} 2^{\frac{d}{2}} S_{d} \int_{0}^{+\infty} F(x+t) t^{\frac{d}{2}} d t,
\end{array}
$$

where $S_{d}$ is the surface of a unit sphere in $d$ dimensions. Eliminating $\lambda$ between the foregoing expressions, we find that the fluid is barotropic, in the sense that $p=p(\rho)$ where the equation of state is entirely specified by $C(f)$. We can now express the free energy (166) as a functional of $\rho$ by writing $F[\rho] \equiv F[f]$. The energy (162) is simply given by

$$
E=\frac{d}{2} \int p d \mathbf{r}+\frac{1}{2} \int \rho \Phi d \mathbf{r} .
$$

On the other hand, the entropy (164) can be written

$$
S=-\frac{2^{\frac{d-2}{2}} S_{d}}{\beta^{d / 2}} \int d \mathbf{r} \int_{0}^{+\infty} C[F(t+\beta \lambda)] t^{\frac{d-2}{2}} d t .
$$

Integrating by parts and using $C^{\prime}[F(x)]=-x$, we find that

$$
S=-\frac{2^{d / 2} S_{d}}{d \beta^{d / 2}} \int d \mathbf{r} \int_{0}^{+\infty} F^{\prime}(t+\beta \lambda)(t+\beta \lambda) t^{d / 2} d t .
$$

Integrating by parts one more time and using Eqs. (277), (278) and (279), we finally obtain

$$
S=\frac{d+2}{2} \beta \int p d \mathbf{r}+\beta \int \lambda \rho d \mathbf{r} .
$$

Collecting all the previous results, the free energy (166) becomes

$$
F[\rho]=-\int \rho\left(\lambda+\frac{p}{\rho}\right) d \mathbf{r}+\frac{1}{2} \int \rho \Phi d \mathbf{r} .
$$

Finally, using the relation $h^{\prime}(x)=-g(x)$ obtained from Eqs. (278) and (279) by a simple integration by parts, it is easy to check that Eq. (277) implies

$$
\lambda^{\prime}(\rho)=-\frac{p^{\prime}(\rho)}{\rho},
$$

so that

$$
\lambda+\frac{p}{\rho}=-\int^{\rho} \frac{p\left(\rho^{\prime}\right)}{\rho^{\prime 2}} d \rho^{\prime} .
$$

Hence, the free energy can be written more explicitly as

$$
F[\rho]=\int \rho \int^{\rho} \frac{p\left(\rho^{\prime}\right)}{\rho^{\prime 2}} d \rho^{\prime} d \mathbf{r}+\frac{1}{2} \int \rho \Phi d \mathbf{r} .
$$




\section{E Equivalence between the stability criteria of the generalized Kramers and Smoluchowski equations}

Let us consider the minimization problem (181). We want to determine the distribution $f_{*}(\mathbf{r}, \mathbf{v})$ which minimizes the free energy $F[f]$ at fixed mass $M[f]=M$. To solve this minimization problem, we can proceed in two steps. First step: we determine the distribution $f_{1}(\mathbf{r}, \mathbf{v})$ which minimizes $F[f]$ at fixed density profile $\rho(\mathbf{r})=\int f d \mathbf{v}$. This gives a distribution $f_{1}[\rho(\mathbf{r}), \mathbf{v}]$ depending on $\rho(\mathbf{r})$ and $\mathbf{v}$. Substituting this distribution in the functional $F[f]$, we obtain a functional $F[\rho] \equiv F\left[f_{1}\right]$ of the density. Second step: we determine the density $\rho_{*}(\mathbf{r})$ which minimizes $F[\rho]$ at fixed mass $M[\rho]=M$. Finally, we have $f_{*}(\mathbf{r}, \mathbf{v})=f_{1}\left[\rho_{*}(\mathbf{r}), \mathbf{v}\right]$.

Let us be more explicit. If we fix the density profile $\rho(\mathbf{r})$, the potential energy $W[\rho]$ is automatically determined. Therefore, minimizing $F[f]=E[f]-T S[f]$ at fixed density profile is equivalent to minimizing $\tilde{F}[f]=$ $K[f]-T S[f]$ at fixed density profile, where $K[f]$ is the kinetic energy. The distribution $f_{1}(\mathbf{r}, \mathbf{v})$ that extremizes $\tilde{F}[f]$ with the constraint $\int f d \mathbf{v}=\rho(\mathbf{r})$ satisfies the first order variations $\delta F+\int \lambda(\mathbf{r}) \delta\left(\int f d \mathbf{v}\right) d \mathbf{r}=0$, where $\lambda(\mathbf{r})$ is a Lagrange multiplier. This leads to

$$
C^{\prime}\left(f_{1}\right)=-\beta\left[\frac{v^{2}}{2}+\lambda(\mathbf{r})\right]
$$

where $\lambda(\mathbf{r})$ is related to $\rho(\mathbf{r})$ by writing $\rho=\int f_{1} d \mathbf{v}$. Since $\delta^{2} F=-T \delta^{2} S=\frac{1}{2} T \int C^{\prime \prime}\left(f_{1}\right)(\delta f)^{2} d \mathbf{r} d \mathbf{v} \geq 0$ the distribution $f_{1}$ is a minimum of $F[f]$ at fixed density profile. Now, we remark that Eq. (288) has the form (274) so that the functional $F[\rho] \equiv F\left[f_{1}\right]$ is explicitly given by

$$
F[\rho]=\int \rho \int^{\rho} \frac{p\left(\rho^{\prime}\right)}{\rho^{\prime 2}} d \rho^{\prime} d \mathbf{r}+\frac{1}{2} \int \rho \Phi d \mathbf{r} .
$$

Therefore, we conclude that $f_{*}(\mathbf{r}, \mathbf{v})=f_{1}\left[\rho_{*}(\mathbf{r}), \mathbf{v}\right]$ is a minimum of $F[f]$ at fixed mass iff $\rho_{*}(\mathbf{r})$ is a minimum of $F[\rho]$ at fixed mass. Thus, the variational problems (181) and (192) are equivalent for global minimization.

We shall now show that they are equivalent for local minimization. A critical point of (181) is a local minimum of $F[f]$ at fixed mass iff inequality (182) is satisfied for all perturbations $\delta f$ that conserve mass. A critical point of (192) is a local minimum of $F[\rho]$ at fixed mass iff inequality (193) is satisfied for all perturbations $\delta \rho$ that conserve mass. In order to make the connection between the second order variations (182) and (193), the idea is to project the perturbation $\delta f$ on a suitable space and write $\delta f=$ $\delta f_{\|}+\delta f_{\perp}$ where $\delta f_{\perp}$ is the orthogonal perturbation (this is a relatively general method that has been applied in different contexts; see 368990 ). We can always write the perturbation in the form

$$
\delta f=\delta f_{\|}+\delta f_{\perp}=\frac{\delta \rho}{\int f^{\prime}(\epsilon) d \mathbf{v}} f^{\prime}(\epsilon)+\delta f_{\perp},
$$

where $\delta f_{\perp} \equiv \delta f-\delta f_{\|}$ensures that all the perturbations are considered. By construction, we have $\int \delta f d \mathbf{v}=$ $\int \delta f_{\|} d \mathbf{v}=\delta \rho$ so that $\int \delta f_{\perp} d \mathbf{v}=0$. Therefore, $\delta f_{\perp}$ is orthogonal to $\delta f_{\|}$in the sense that

$$
\int \delta f_{\|} \delta f_{\perp} \frac{1}{f^{\prime}(\epsilon)} d \mathbf{v} \propto \int \delta f_{\perp} d \mathbf{v}=0 .
$$

Then, we readily obtain

$$
\begin{aligned}
\int \frac{(\delta f)^{2}}{f^{\prime}(\epsilon)} d \mathbf{v} & =\int \frac{\left(\delta f_{\perp}\right)^{2}}{f^{\prime}(\epsilon)} d \mathbf{v}+\int \frac{\left(\delta f_{\|}\right)^{2}}{f^{\prime}(\epsilon)} d \mathbf{v} \\
& =\int \frac{\left(\delta f_{\perp}\right)^{2}}{f^{\prime}(\epsilon)} d \mathbf{v}+\frac{(\delta \rho)^{2}}{\int f^{\prime}(\epsilon) d \mathbf{v}}
\end{aligned}
$$

Now, a critical point of (181) is of the form $f=f(\epsilon)$ with $\epsilon=v^{2} / 2+\Phi(\mathbf{r})$ and $f^{\prime}(\epsilon)<0$. This implies that $\rho=\int f(\epsilon) d \mathbf{v}=\rho(\Phi)$ and $\rho^{\prime}(\Phi)=\int f^{\prime}(\epsilon) d \mathbf{v}$. Therefore, Eq. (292) can be rewritten

$$
\int \frac{(\delta f)^{2}}{f^{\prime}(\epsilon)} d \mathbf{v}=\int \frac{\left(\delta f_{\perp}\right)^{2}}{f^{\prime}(\epsilon)} d \mathbf{v}+\frac{(\delta \rho)^{2}}{\rho^{\prime}(\Phi)} .
$$

Combining Eqs. (293), (182) and (193) we finally obtain

$$
\delta^{2} F[\delta f]=-\frac{1}{2} \int \frac{\left(\delta f_{\perp}\right)^{2}}{f^{\prime}(\epsilon)} d \mathbf{r} d \mathbf{v}+\delta^{2} F[\delta \rho] .
$$

If $\delta^{2} F[\delta \rho] \geq 0$ for all perturbations $\delta \rho$ that conserve mass, then $\delta^{2} F[\delta f] \geq 0$ for all perturbations $\delta f$ that conserve mass. Alternatively, if there exists a perturbation $\delta \rho_{*}$ such that $\delta^{2} F\left[\delta \rho_{*}\right]<0$, by taking $\delta f_{*}$ in the form (290) with $\delta \rho=\delta \rho_{*}$ and $\delta f_{\perp}=0$, we get $\delta^{2} F\left[\delta f_{*}\right]=\delta^{2} F\left[\delta \rho_{*}\right]<0$. We conclude that $f(\mathbf{r}, \mathbf{v})$ is a local minimum of $F[f]$ at fixed $M$ iff $\rho(\mathbf{r})$ is a local minimum of $F[\rho]$ at fixed $M$. Thus: (181) $\Leftrightarrow$ (192) for local and global minimizations.

There are several consequences to this result (see also the more detailed discussion in 90):

(i) We have seen that $f$ is a linearly dynamically stable steady state of the generalized Kramers equation (159) iff it is a (local) minimum of $F[f]$ at fixed mass $M[f]=M$. On the other hand, we have seen that $\rho$ is a linearly dynamically stable steady state of the generalized Smoluchowski equation (44) iff it is a (local) minimum of $F[\rho]$ at fixed mass $M[\rho]=M$. According to the above-mentioned result (181) $\Leftrightarrow$ (192), we conclude that: $f_{*}(\mathbf{r}, \mathbf{v})=f_{1}\left[\rho_{*}(\mathbf{r}), \mathbf{v}\right]$ is a linearly dynamically stable steady state of the generalized Kramers equation iff $\rho_{*}(\mathbf{r})$ is a linearly dynamically stable steady state of the generalized Smoluchowski equation.

(ii) It can be shown that a distribution function which minimizes a functional of the form $F[f]=E[f]-T S[f]$ (where $T$ is a constant and $S[f]$ is given by Eq. (164) where $C(f)$ is an arbitrary convex function) at fixed mass $M[f]=M$ is a nonlinearly 8 dynamically stable stationary solution of the Vlasov equation (see, e.g., the case of stellar systems 92]). Therefore, the generalized mean field Kramers equation (159), which precisely solves this minimization problem, can be used as a numerical algorithm

\footnotetext{
8 We implicitly consider here the formal nonlinear dynamical stability in the sense of Holm et al. 91.
} 
to construct nonlinearly dynamically stable stationary solutions of the Vlasov equation. Indeed, if a distribution is linearly dynamically stable with respect to the generalized Kramers equation then it is nonlinearly dynamically stable with respect to the Vlasov equation (but the converse may be wrong, see below).

(iii) It can be shown that a density profile is a nonlinearly dynamically stable stationary solution of the Euler equation with a barotropic equation of state iff it minimizes a functional of the form $F[\rho]$ (where $F[\rho]$ is given by Eq. (47)) at fixed mass $M[\rho]=M$ (see, e.g., the case of barotropic stars 92]). Therefore, the generalized mean field Smoluchowski equation (44), which precisely solves this minimization problem, can be used as a numerical algorithm to construct nonlinearly dynamically stable stationary solutions of the Euler equation. Indeed, a density profile is linearly dynamically stable with respect to the generalized Smoluchowski equation iff it is nonlinearly dynamically stable with respect to the barotropic Euler equation.

(iv) According to the above mentioned results, a distribution function $f=f(\epsilon)$ with $f^{\prime}(\epsilon)<0$ is a nonlinearly dynamically stable stationary solution of the Vlasov equation if the corresponding barotropic gas with equation of state $p=p(\rho)$ is a nonlinearly dynamically stable stationary solution of the Euler equation. In astrophysics, this corresponds to the nonlinear Antonov first law 92 . However, the minimization of $F[f]=E[f]-T S[f]$ at fixed mass $M[f]=M$ (problem with one constraint) is just a sufficient condition of nonlinear dynamical stability with respect to the Vlasov equation. Thus, this minimization problem does not allow to construct all the nonlinearly dynamically stable stationary solutions of the Vlasov equation. A larger class of nonlinearly dynamically stable stationary solutions is obtained by maximizing a Casimir functional of the form $S[f]$ (where $S[f]$ is given by Eq. (164)) at fixed mass $M[f]=M$ and energy $E[f]=E$ (problem with two constraints) 92. A numerical algorithm solving this maximization problem is proposed in [33. In case of "ensemble inequivalence", these solutions cannot be obtained by minimizing $F[f]=E[f]-T S[f]$ at fixed mass $M[f]=M$. Therefore, the problem with two constraints ("microcanonical") provides a refined condition of nonlinear dynamical stability with respect to the problem with one constraint ("canonical").

\section{F Extension of the Eddington formula}

In Sec. 4.7, we have seen that a distribution function $f=$ $f(\epsilon)$ determines a barotropic equation of state $p=p(\rho)$ and we have explained how to obtain it (some explicit examples have been given in Sec. 5). We shall now consider the inverse problem: find the distribution function $f=f(\epsilon)$ leading to the equation of state $p(\rho)$. This problem was first encountered in astrophysics and solved by Eddington 93. In astrophysics, a distribution function of the form $f=f(\epsilon)$ describes a particular class of spherical stellar systems that are stationary solutions of the VlasovPoisson system. To any such stellar system, we can asso- ciate a corresponding barotropic star with an equation of state $p=p(\rho)$ which is a stationary solution of the EulerPoisson system. The problem is to find the equation of state $p=p(\rho)$ corresponding to the distribution function $f=f(\epsilon)$ and vice et versa. A similar problem arises in the context of nonlinear mean field Fokker-Planck equations. Thus, we can adapt many results of astrophysics to the present situation. For sake of generality, we shall consider the Eddington inverse problem in a space of arbitrary dimension $d$.

Knowing the equation of state $p=p(\rho)$, we can obtain the equilibrium density $\rho(\Phi)$ by integrating the condition of hydrostatic equilibrium (190). The problem now is to determine $f(\epsilon)$ from the knowledge of $\rho(\Phi)$. Let us rewrite the density in the form

$$
\rho(\Phi)=\int_{0}^{+\infty} f(\epsilon) S_{d} v^{d-1} d v
$$

We shall consider two cases:

(i) We first assume that the distribution function has a compact support so that $f=0$ if $\epsilon \geq \epsilon_{m}$. In that case, the range of integration in Eq. (295) is restricted to $v \leq$ $\sqrt{2\left(\epsilon_{m}-\Phi\right)}$ so that

$$
\rho(\Phi)=\int_{0}^{\sqrt{2\left(\epsilon_{m}-\Phi\right)}} f(\epsilon) S_{d} v^{d-1} d v .
$$

Taking $\epsilon=\frac{v^{2}}{2}+\Phi$ as a variable of integration instead of $v$ we obtain

$$
\rho(\Phi)=\int_{\Phi}^{\epsilon_{m}} f(\epsilon) S_{d}[2(\epsilon-\Phi)]^{\frac{d-2}{2}} d \epsilon .
$$

It is convenient at this stage to define $\psi=\epsilon_{m}-\Phi$ and $x=\epsilon_{m}-\epsilon$. In terms of these variables, we get

$$
\rho(\psi)=2^{\frac{d-2}{2}} S_{d} \int_{0}^{\psi} f(x)(\psi-x)^{\frac{d-2}{2}} d x .
$$

In $d=3$, taking the derivative of Eq. 2298) we find

$$
\frac{1}{\sqrt{8} \pi} \frac{d \rho}{d \psi}=\int_{0}^{\psi} \frac{f(x)}{\sqrt{\psi-x}} d x .
$$

This is an Abel integral whose solution is

$$
\begin{array}{r}
f(x)=\frac{1}{\sqrt{8} \pi^{2}} \frac{d}{d x} \int_{0}^{x} \frac{d \rho}{d \psi} \frac{d \psi}{\sqrt{x-\psi}} \\
=\frac{1}{\sqrt{8} \pi^{2}}\left[\int_{0}^{x} \frac{d^{2} \rho}{d \psi^{2}} \frac{d \psi}{\sqrt{x-\psi}}+\frac{1}{\sqrt{x}}\left(\frac{d \rho}{d \psi}\right)_{\psi=0}\right] .
\end{array}
$$

This is the Eddington formula 93. In $d=1$, Eq. (298) becomes

$$
\frac{1}{\sqrt{2}} \rho(\psi)=\int_{0}^{\psi} \frac{f(x)}{\sqrt{\psi-x}} d x .
$$


Comparing with the previous case, we immediately have

$$
\begin{array}{r}
f(x)=\frac{1}{\sqrt{2} \pi} \frac{d}{d x} \int_{0}^{x} \rho(\psi) \frac{d \psi}{\sqrt{x-\psi}} \\
=\frac{1}{\sqrt{2} \pi}\left[\int_{0}^{x} \frac{d \rho}{d \psi} \frac{d \psi}{\sqrt{x-\psi}}+\frac{1}{\sqrt{x}} \rho(\psi=0)\right] .
\end{array}
$$

Finally, in $d=2$, Eq. (297) reduces to

$$
\rho(\Phi)=2 \pi \int_{\Phi}^{\epsilon_{m}} f(\epsilon) d \epsilon,
$$

and we get the very simple result

$$
f(\epsilon)=-\frac{1}{2 \pi} \frac{d \rho}{d \Phi}(\epsilon)
$$

(ii) We now consider the case where the distribution function takes strictly positive values for all energies so that $\epsilon_{m} \rightarrow+\infty$. Then Eq. (295) becomes

$$
\rho(\Phi)=\int_{\Phi}^{+\infty} f(\epsilon) S_{d}[2(\epsilon-\Phi)]^{\frac{d-2}{2}} d \epsilon .
$$

In $d=3$, taking the derivative of Eq. (305) we obtain

$$
-\frac{1}{\sqrt{8} \pi} \frac{d \rho}{d \Phi}=\int_{\Phi}^{+\infty} \frac{f(\epsilon)}{\sqrt{\epsilon-\Phi}} d \epsilon .
$$

This is an Abel integral whose solution is

$$
\begin{array}{r}
f(\epsilon)=\frac{1}{\sqrt{8} \pi^{2}} \frac{d}{d \epsilon} \int_{\epsilon}^{+\infty} \frac{d \rho}{d \Phi} \frac{d \Phi}{\sqrt{\Phi-\epsilon}} \\
=\frac{1}{\sqrt{8} \pi^{2}}\left[\int_{\epsilon}^{+\infty} \frac{d^{2} \rho}{d \Phi^{2}} \frac{d \Phi}{\sqrt{\Phi-\epsilon}}-\lim _{\Phi \rightarrow+\infty} \frac{\frac{d \rho}{d \Phi}}{\sqrt{\Phi-\epsilon}}\right] .
\end{array}
$$

In $d=1$, Eq. (305) becomes

$$
\frac{1}{\sqrt{2}} \rho(\Phi)=\int_{\Phi}^{+\infty} \frac{f(\epsilon)}{\sqrt{\epsilon-\Phi}} d \epsilon
$$

and we get

$$
\begin{array}{r}
f(\epsilon)=-\frac{1}{\sqrt{2} \pi} \frac{d}{d \epsilon} \int_{\epsilon}^{+\infty} \rho(\Phi) \frac{d \Phi}{\sqrt{\Phi-\epsilon}} \\
=-\frac{1}{\sqrt{2} \pi}\left[\int_{\epsilon}^{+\infty} \frac{d \rho}{d \Phi} \frac{d \Phi}{\sqrt{\Phi-\epsilon}}-\lim _{\Phi \rightarrow+\infty} \frac{\rho}{\sqrt{\Phi-\epsilon}}\right] .
\end{array}
$$

For $d=2$, Eq. (304) remains unchanged. For example, the distribution function associated with the Fermi-Dirac statistics in physical space

$$
\rho(\Phi)=\frac{\sigma_{0}}{1+e^{\beta \Phi+\alpha}}
$$

is

$$
f(\epsilon)=\frac{\sigma_{0} \beta}{8 \pi \cosh ^{2}\left[\frac{1}{2}(\beta \epsilon+\alpha)\right]}, \quad(d=2) .
$$

\section{G Derivation of the $H$-theorems}

Let us derive the $H$-theorem (24) for the NFP equation (8) -(2). The time variations of the entropy (21) and of the energy (17) associated with an external potential are

$$
\dot{S}=-\int C^{\prime}(\rho) \frac{\partial \rho}{\partial t} d \mathbf{r}, \quad \dot{E}=\int \Phi_{e x t} \frac{\partial \rho}{\partial t} d \mathbf{r}
$$

On the other hand, using Eqs. (18) and (2), the energy associated with a binary potential of interaction can be written

$$
E=\frac{1}{2} \int \rho(\mathbf{r}, t) u\left(\left|\mathbf{r}-\mathbf{r}^{\prime}\right|\right) \rho\left(\mathbf{r}^{\prime}, t\right) d \mathbf{r} d \mathbf{r}^{\prime} .
$$

Its time derivative is

$$
\begin{aligned}
\dot{E} & =\frac{1}{2} \int \frac{\partial \rho}{\partial t}(\mathbf{r}, t) u\left(\left|\mathbf{r}-\mathbf{r}^{\prime}\right|\right) \rho\left(\mathbf{r}^{\prime}, t\right) d \mathbf{r} d \mathbf{r}^{\prime} \\
& +\frac{1}{2} \int \rho(\mathbf{r}, t) u\left(\left|\mathbf{r}-\mathbf{r}^{\prime}\right|\right) \frac{\partial \rho}{\partial t}\left(\mathbf{r}^{\prime}, t\right) d \mathbf{r} d \mathbf{r}^{\prime}
\end{aligned}
$$

Interchanging the dummy variables $\mathbf{r}$ and $\mathbf{r}^{\prime}$ and using Eq. (2), we finally obtain

$$
\dot{E}=\int \Phi \frac{\partial \rho}{\partial t} d \mathbf{r}
$$

Therefore, the time variation of the free energy (23) is given by

$$
\dot{F}=\dot{E}-T \dot{S}=\int\left(\Phi+T C^{\prime}(\rho)\right) \frac{\partial \rho}{\partial t} d \mathbf{r} .
$$

Using Eq. (9) and integrating by parts, we obtain

$$
\dot{F}=\int \mathbf{J} \cdot\left(\nabla \Phi+T C^{\prime \prime}(\rho) \nabla \rho\right) d \mathbf{r} .
$$

Inserting Eqs. (10), (20) and (22) in Eq. (317), we finally obtain the $H$-theorem (24).

Let us derive the $H$-theorem (25) for the NFP equation (8)-(3). The time variation of the energy given by Eq. (19) is

$$
\begin{aligned}
\dot{E}=\frac{1}{\lambda} \int( & \left.\nabla \Phi \cdot \nabla \frac{\partial \Phi}{\partial t}+k^{2} \Phi \frac{\partial \Phi}{\partial t}\right) d \mathbf{r} \\
& +\int \frac{\partial \rho}{\partial t} \Phi d \mathbf{r}+\int \rho \frac{\partial \Phi}{\partial t} d \mathbf{r}
\end{aligned}
$$

Integrating the first term by parts and using Eq. (3), it can be rewritten

$$
\dot{E}=-\frac{1}{\lambda \epsilon} \int\left(\Delta \Phi-k^{2} \Phi-\lambda \rho\right)^{2} d \mathbf{r}+\int \frac{\partial \rho}{\partial t} \Phi d \mathbf{r} .
$$

Inserting Eq. (8) and proceeding as above, we obtain the $H$-theorem (25).

Consider now the generalized Smoluchowski equation (44). The time variation of the free energy (47) is

$$
\dot{F}=\int\left(\Phi+\int^{\rho} \frac{p\left(\rho^{\prime}\right)}{\rho^{\prime 2}} d \rho^{\prime} d \mathbf{r}+\frac{p}{\rho}\right) \frac{\partial \rho}{\partial t} d \mathbf{r}
$$


Inserting Eq. (44) in Eq. (320) and integrating by parts we obtain the $H$-theorem (48).

Let us derive the $H$-theorem (168) for the NFP equation (159)-(2). The time variations of the entropy (164) and of the energy (162) are

$$
\dot{S}=-\int C^{\prime}(f) \frac{\partial f}{\partial t} d \mathbf{r} d \mathbf{v}, \quad \dot{E}=\int\left(\frac{v^{2}}{2}+\Phi\right) \frac{\partial f}{\partial t} d \mathbf{r} d \mathbf{v} .
$$

For $D=\xi=0$, the NFP equation (159)-(2) reduces to the Vlasov equation. The Vlasov equation conserves the energy and the Casimirs. Indeed, using integrations by parts, we have

$$
\begin{gathered}
\dot{E}=\int\left(\frac{v^{2}}{2}+\Phi\right)\left(-\mathbf{v} \cdot \frac{\partial f}{\partial \mathbf{r}}+\nabla \Phi \cdot \frac{\partial f}{\partial \mathbf{v}}\right) d \mathbf{r} d \mathbf{v} \\
=\int(\nabla \Phi \cdot \mathbf{v}-\mathbf{v} \cdot \nabla \Phi) f d \mathbf{r} d \mathbf{v}=0
\end{gathered}
$$

and

$$
\begin{array}{r}
\dot{S}=-\int C^{\prime}(f)\left(-\mathbf{v} \cdot \frac{\partial f}{\partial \mathbf{r}}+\nabla \Phi \cdot \frac{\partial f}{\partial \mathbf{v}}\right) d \mathbf{r} d \mathbf{v} \\
=\int\left[\mathbf{v} \cdot \frac{\partial C(f)}{\partial \mathbf{r}}-\nabla \Phi \cdot \frac{\partial C(f)}{\partial \mathbf{v}}\right] d \mathbf{r} d \mathbf{v} \\
=\int\left[\frac{\partial}{\partial \mathbf{r}} \cdot(C(f) \mathbf{v})-\frac{\partial}{\partial \mathbf{v}} \cdot(C(f) \nabla \Phi)\right] d \mathbf{r} d \mathbf{v}=0
\end{array}
$$

Therefore, coming back to the NFP equation (159)-(2), the only contribution to the time variation of the free energy (166) comes from the Fokker-Planck current. Using Eqs. (321) and (160), we have

$$
\begin{aligned}
& \dot{F}=\int\left(\frac{v^{2}}{2}+\Phi+T C^{\prime}(f)\right) \frac{\partial f}{\partial t} d \mathbf{r} d \mathbf{v} \\
& =-\int\left(\frac{v^{2}}{2}+\Phi+T C^{\prime}(f)\right) \cdot \frac{\partial \mathbf{J}}{\partial \mathbf{v}} d \mathbf{r} d \mathbf{v} \\
& =\int\left(\mathbf{v}+T C^{\prime \prime}(f) \frac{\partial f}{\partial \mathbf{v}}\right) \cdot \mathbf{J} d \mathbf{r} d \mathbf{v} .
\end{aligned}
$$

Inserting Eqs. (161), (163) and (165) in Eq. (324) and integrating by parts we obtain the $H$-theorem (168).

Let us finally derive the $H$-theorem (214) for the damped Euler equations (211), (212) and (2). The time variation of the free energy (213) is

$$
\begin{aligned}
\dot{F}=\int\left(\Phi+\int \frac{p\left(\rho^{\prime}\right)}{\rho^{\prime 2}} d \rho^{\prime} d \mathbf{r}\right. & \left.+\frac{p}{\rho}+\frac{\mathbf{u}^{2}}{2}\right) \frac{\partial \rho}{\partial t} d \mathbf{r} \\
& +\int \rho \mathbf{u} \cdot \frac{\partial \mathbf{u}}{\partial t} d \mathbf{r}
\end{aligned}
$$

Substituting Eqs. (211) and (212) in Eq. (325) and integrating by parts, we get

$$
\dot{F}=\int \rho \mathbf{u} \cdot\left[\nabla\left(\frac{\mathbf{u}^{2}}{2}\right)-\xi \mathbf{u}-(\mathbf{u} \cdot \nabla) \mathbf{u}\right] d \mathbf{r} .
$$

Using $(\mathbf{u} \cdot \nabla) \mathbf{u}=\nabla\left(\mathbf{u}^{2} / 2\right)-\mathbf{u} \times(\nabla \times \mathbf{u})$ we finally obtain the result (214).

\section{H Polytropic equation of state}

For $n>d / 2$ (case 1$)$, the polytropic DF can we written

$$
f=A\left(\epsilon_{m}-\epsilon\right)_{+}^{n-d / 2} .
$$

The density and the pressure can be expressed as

$$
\rho=A S_{d} Q_{0}(\Phi), \quad p=\frac{1}{d} A S_{d} Q_{2}(\Phi),
$$

with

$$
Q_{k}=\int_{0}^{\sqrt{2\left(\epsilon_{m}-\Phi\right)}}\left(\epsilon_{m}-\Phi-\frac{v^{2}}{2}\right)^{n-d / 2} v^{k+d-1} d v
$$

Setting $x=v^{2} /\left[2\left(\epsilon_{m}-\Phi\right)\right]$, we obtain

$$
\begin{aligned}
& Q_{k}=2^{(k+d-2) / 2}\left(\epsilon_{m}-\Phi\right)^{n+k / 2} \\
& \times \int_{0}^{1}(1-x)^{n-d / 2} x^{(k+d-2) / 2} d x .
\end{aligned}
$$

The integral can be expressed in terms of Gamma functions leading to

$$
\begin{aligned}
& Q_{k}=2^{(k+d-2) / 2}\left(\epsilon_{m}-\Phi\right)^{n+k / 2} \\
& \times \frac{\Gamma((d+k) / 2) \Gamma(1-d / 2+n)}{\Gamma(1+k / 2+n)} .
\end{aligned}
$$

Then, the density and the pressure can be expressed in terms of the potential $\Phi$ as

$$
\rho=A S_{d}\left(\epsilon_{m}-\Phi\right)^{n} 2^{d / 2-1} \frac{\Gamma(d / 2) \Gamma(1-d / 2+n)}{\Gamma(1+n)},
$$

$p=\frac{A S_{d}}{n+1}\left(\epsilon_{m}-\Phi\right)^{n+1} 2^{d / 2-1} \frac{\Gamma(d / 2) \Gamma(1-d / 2+n)}{\Gamma(1+n)}$,

where we have used the identity $\Gamma(n+1)=n \Gamma(n)$ to simplify the second expression. Eliminating the potential $\Phi$ between these equations, we obtain the polytropic equation of state (231) with $K$ given by Eq. (232).

For $n<-1$ (case 2), the polytropic DF can we written

$$
f=A\left(\epsilon_{m}+\epsilon\right)^{n-d / 2} .
$$

The density and the pressure can be expressed as

$$
\rho=A S_{d} R_{0}(\Phi), \quad p=\frac{1}{d} A S_{d} R_{2}(\Phi),
$$

with

$$
R_{k}=\int_{0}^{+\infty}\left(\epsilon_{m}+\Phi+\frac{v^{2}}{2}\right)^{n-d / 2} v^{k+d-1} d v
$$

Setting $x=v^{2} /\left[2\left(\epsilon_{m}+\Phi\right)\right]$, we obtain

$$
\begin{aligned}
& R_{k}=2^{(k+d-2) / 2}\left(\epsilon_{m}+\Phi\right)^{n+k / 2} \\
\times & \int_{0}^{+\infty}(1+x)^{n-d / 2} x^{(k+d-2) / 2} d x .
\end{aligned}
$$


The integral can be expressed in terms of Gamma functions leading to

$$
\begin{array}{r}
R_{k}=2^{(k+d-2) / 2}\left(\epsilon_{m}+\Phi\right)^{n+k / 2} \\
\times \frac{\Gamma((d+k) / 2) \Gamma(-k / 2-n)}{\Gamma(d / 2-n)} .
\end{array}
$$

Then, the density and the pressure can be expressed in terms of the potential $\Phi$ as

$$
\begin{gathered}
\rho=A S_{d}\left(\epsilon_{m}+\Phi\right)^{n} 2^{d / 2-1} \frac{\Gamma(d / 2) \Gamma(-n)}{\Gamma(d / 2-n)} \\
p=-\frac{A S_{d}}{n+1}\left(\epsilon_{m}+\Phi\right)^{n+1} 2^{d / 2-1} \frac{\Gamma(d / 2) \Gamma(-n)}{\Gamma(d / 2-n)}
\end{gathered}
$$

where we have used the identity $\Gamma(n+1)=n \Gamma(n)$ to simplify the second expression. Eliminating the potential $\Phi$ between these equations, we obtain the polytropic equation of state (231) with $K$ given by Eq. (233).

Note, as a final remark, that spatially homogeneous polytropic distributions are obtained by taking $\Phi(\mathbf{r})=0$ in the above expressions.

\section{References}

1. A.D. Fokker, Ann. Physik 43, 810 (1914).

2. M. Planck, Sitzber. Preuss. Akad. Wiss., p. 324 (1917).

3. A. Einstein, Ann. Physik 17, 549 (1905).

4. M. von Smoluchowski, Ann. Physik 48, 1103 (1915).

5. H.A. Kramers, Physica A 7, 284 (1940).

6. O. Klein, Arkiv for Mathematik, Astronomi, och Fysik 16, No 5 (1921).

7. H. Risken, The Fokker-Planck equation (Springer, 1989).

8. P.H. Chavanis, C. R. Physique 7, 318 (2006).

9. C. Tsallis, J. Stat. Phys. 52, 479 (1988).

10. S. Abe, Phys. Lett. A 224, 326 (1997).

11. E.P. Borges, I. Roditi, Phys. Lett. A 246, 399 (1998).

12. G. Kaniadakis, Physica A 296, 405 (2001).

13. J. Naudts, Physica A 340, 32 (2004).

14. G. Kaniadakis, M. Lissia, A.M. Scarfone, Phys. Rev. E 71, 046128 (2005).

15. J. Harvda, F. Charvat, Kybernetica 3, 30 (1967).

16. D.P. Mittal, Metrika 22, 35 (1975).

17. B.D. Sharma, I.J. Taneja, Metrika 22, 205 (1975).

18. A. Reyni, Probability Theory (North-Holland, Amsterdam, 1970).

19. B.D. Sharma, D.P. Mittal, J. Math. Sci. 1, 28 (1975).

20. G. Kaniadakis, M. Lissia, Physica A 340, xv-xix (2004).

21. A.R. Plastino, A. Plastino, Physica A 222, 347 (1995).

22. H. Spohn, J. Physique 3, 69 (1993).

23. C. Tsallis, D.J. Bukman, Phys. Rev. E 54, R2197 (1996).

24. D. Stariolo, Phys. Rev. E 55, 4806 (1997).

25. L. Borland, Phys. Rev. E 57, 6634 (1998).

26. F. Nobre, E. Curado, G. Rowlands, Physica A 334, 109 (2004).

27. G. Kaniadakis, P. Quarati Phys. Rev. E 49, 5103 (1994).

28. P.H. Chavanis, J. Sommeria, R. Robert, Astrophys. J. 471, 385 (1996).
29. D. Lynden-Bell, Mon. Not. Roy. Astr. Soc. 136, 101 (1967).

30. J. Sopik, C. Sire, P.H. Chavanis, Phys. Rev. E 74, 011112 (2006).

31. S. Martinez, A.R. Plastino, A. Plastino, Physica A 259, 183 (1998).

32. T.D. Frank, Physica A 310, 397 (2002).

33. P.H. Chavanis, Phys. Rev. E 68, 036108 (2003).

34. P.H. Chavanis, Physica A 332, 89 (2004).

35. E. Curado, F. Nobre, Phys. Rev. E 67, 021107 (2003).

36. T.D. Frank, Nonlinear Fokker-Planck Equations: Fundamentals and Applications (Springer-Verlag, 2005).

37. Dynamics and thermodynamics of systems with long range interactions, edited by Dauxois, T., Ruffo, S., Arimondo, E. and Wilkens, M. Lect. Not. in Phys. 602 (Springer, 2002)

38. Y. Kuramoto, Chemical oscillations, waves, and turbulence (Springer, Berlin, 1984).

39. N. Martzel, C. Aslangul, J. Phys. A 34, 11225 (2001).

40. P.H. Chavanis, Physica A 361, 55 (2006); Physica A 361, 81 (2006).

41. P.H. Chavanis, C. Rosier, C. Sire, Phys. Rev. E 66, 036105 (2002).

42. C. Sire, P.H. Chavanis, Phys. Rev. E 66, 046133 (2002).

43. P.H. Chavanis, J. Vatteville, F. Bouchet, Eur. Phys. J. B 46, 61 (2005).

44. M. Antoni, S. Ruffo, Phys. Rev. E 52, 2361 (1995).

45. P.H. Chavanis, C. Sire, Phys. Rev. E 69, 016116 (2004).

46. M. Shiino, Phys. Rev. E 67, 056118 (2003).

47. J.D. Murray, Mathematical Biology (Springer, Berlin, 1991).

48. C.S. Patlak, Bull. of Math. Biophys. 15, 311 (1953).

49. E.F. Keller, L.A. Segel, J. Theor. Biol. 30, 225 (1971).

50. H. Othmer, A. Stevens, SIAM J. Appl. Math. 57, 1044 (1997).

51. P.H. Chavanis, C. Sire, Physica A 384, 199 (2007).

52. D. Horstmann, From 1970 until present: the Keller-Segel model in chemotaxis and its consequences, Jahresber. Deutsch. Math. Verein. 106, 51 (2004).

53. P.H. Chavanis, C. Sire, Physica A 387, 1999 (2008).

54. P.H. Chavanis, P. Laurençot, M. Lemou, Physica A 341, 145 (2004).

55. P.H. Chavanis, arXiv:0803.0263

56. C. Sire, P.H. Chavanis, Phys. Rev. E 69, 066109 (2004).

57. P.H. Chavanis, C. Sire, Phys. Rev. E 70, 026115 (2004).

58. P.H. Chavanis, C. Sire, Phys. Rev. E 73, 066103 (2006).

59. P.H. Chavanis, C. Sire, Phys. Rev. E 73, 066104 (2006).

60. P. Debye, E. Hückel, Phys. Z. 24, 305 (1923).

61. R. Robert, J. Sommeria, Phys. Rev. Lett. 69, 2776 (1992).

62. P.H. Chavanis, Physica D 200, 257 (2005).

63. J.M. Burgers, The Nonlinear Diffusion Equation (Riedel, Boston, 1974).

64. A. Stevens, SIAM J. Appl. Math. 61, 183 (2000).

65. T.J. Newman, R. Grima, Phys. Rev. E 70, 051916 (2004).

66. P.H. Chavanis, M. Ribot, C. Rosier, C. Sire, Banach Center Publ. 66, 103 (2004)

67. P.H. Chavanis, C. Sire, Physica A 384, 199 (2007).

68. R. Grima, Curr. Topics. Dev. Bio 81, 435 (2008).

69. P.H. Chavanis, Physica A 359, 177 (2006).

70. D.E. McLaughlin, R.E. Pudritz, Astrophys. J. 476, 750 (1997).

71. P.H. Chavanis, C. Sire, Physica A 375, 140 (2007).

72. T. Hillen, K. Painter, Adv. Appl. Math. 26, 280 (2001). 
73. P.H. Chavanis, Eur. Phys. J. B 54, 525 (2006).

74. P.H. Chavanis, Banach Center Publ. 66, 79 (2004).

75. J. Paret, P. Tabeling, Phys. Fluids 10, 3126 (1998).

76. M. Rivera, X.L. Wu, Phys. Rev. Lett. 85, 976 (2000).

77. P.H. Chavanis, A\&A 356, 1089 (2000).

78. A. Gamba, D. Ambrosi, A. Coniglio, A. de Candia, S. di Talia, E. Giraudo, G. Serini, L. Preziosi, F. Bussolino, Phys. Rev. Lett. 90, 118101 (2003).

79. F. Filbet, P. Laurençot, B. Perthame, J. Math. Biol. 50, 189 (2005).

80. P.H. Chavanis, C. Sire, Physica A 356, 419 (2005).

81. Du Jiulin, Astrophys. Space Sci. 306, 247 (2006).

82. P.H. Chavanis, Phys. Rev. E 69, 066126 (2004).

83. P. Biler, P. Laurençot, T. Nadzieja, Adv. Differential Equations 9, 563 (2004).

84. A. Einstein, Ann. Physik 33, 1275 (1910).

85. E.G.D. Cohen, Physica A 305, 19 (2002).

86. J. Dolbeault, P. Markowich, D. Ölz, C. Schmeiser, Arch. Rational Mech. Anal. 1, 133 (2007).

87. P.H. Chavanis, C. Sire, arXiv:0708.3163

88. P.H. Chavanis, Eur. Phys. J. B 52, 433 (2006).

89. F. Bouchet, arXiv:0710.5094

90. P.H. Chavanis, AIP Conf. Proc. 970, 39 (2008).

91. D.D. Holm, J.E. Marsden, T. Ratiu, A. Weinstein, Phys. Rep. 123, 1 (1985).

92. P.H. Chavanis, A\&A 451, 109 (2006).

93. A.S. Eddington, MNRAS 76, 572 (1916). 